\title{
Testing the fractional integration parameter revisited: a Fractional Dickey-Fuller Test
}

\author{
Ahmed BENSALMA 1 and Mohamed BENTARZI 2
}

\begin{abstract}
In this paper, in the first step, we show that the fractional DickeyFuller test proposed by Dolado et al [10] is useless in practice. In the second step, we propose a new testing procedure for the degree of fractional integration of a time series inspired on the unit root test of Dickey-Fuller [7]. The composite null hypothesis is that of $d \geq d_{0}$ against $d<d_{0}$. The test statistics is the same as in Dickey-Fuller test using as output $(1-L)^{d_{0}} y_{t}$ instead of $(1-L) y_{t}$ and as input $(1-L)^{-1+d_{0}} y_{t-1}$ and eventually some lag of $(1-L)^{d_{0}} y_{t}$ instead some lag of $(1-L) y_{t}$, exploiting the fact that if $y_{t}$ is $I(d)$ then $\Delta^{-1+d_{0}} y_{t}$ is $I(1)$ under the null $d=d_{0}$. If $d \geq d_{0}$, using the generalization of Sowell's result [23], we propose a test based on the least favorable case, $d=d_{0}$, to control type $I$ error, and when $d<d_{0}$ we show that the tests statistics diverges to $-\infty$, providing consistency. Through a simulation study, we show the good performance of the test in terms of size and power. Finally, in order to show how to use the new testing procedure, the test is applied to the well-known Nelson and Plosser data.
\end{abstract}

Keywords: Fractional integration, Fractional unit root; Dickey-Fuller unit root test; Fractional Dickey-Fuller test.

\section{Introduction}

As the most popular long memory model and a useful extension of the classical ARIMA models, the fractionally integrated autoregressive moving average (ARFIMA) process, introduced by Granger and Jojeux [11] and Hosking [13], has seen a considerable interest in the past three decades and has been widely applied in many fields like hydrology, economics and finance. The ARFIMA process generalizes the standard linear $\operatorname{ARIMA}(p, d, q)$ model by permitting to the degree of integration $d$ to be non-integer. Compared with the standard $A R M A$ and ARIMA specifications, the ARFIMA generalization provides a more flexible framework in modelling the long range dependence, where a special role is played by the fractional differencing parameter $d$ whose precise determination is very important in applied work.

\footnotetext{
${ }^{1}$ High national school of statistic and applied economic (ENSSEA), Algiers, Algeria, Email: bensalma.ahmed@gmail.com

2 Faculty of Mathematics, University of Science and Technology Houari Boumediene, Algiers, Algeria. , Email: bentarzimohamed@yahoo.fr
} 
The stationary and invertible Fractional $\operatorname{ARIMA}(p, d, q)$ processes, is defined as the following

$$
\left(1-\sum_{i=1}^{p} \phi_{i} L^{i}\right)(1-L)^{d} y_{t}=\left(1-\sum_{i=1}^{q} \theta_{i} L^{i}\right) u_{t}, \quad t \in \mathbb{Z}, \quad-0.5<d<0.5,
$$

where $L$ is the backshift operator and $u_{t}$ are independently and identically distributed (i.i.d) random variables with zero mean and finite variance; $\left(1-\sum_{i=1}^{p} \phi_{i} L^{i}\right)$ and $\left(1-\sum_{i=1}^{q} \theta_{i} L^{i}\right)$ are polynomial functions of $L$ with order $p$ and $q$, and both of them have only roots outside the unit circle. The fractional difference operator $(1-L)^{d}$ is defined by its Maclaurin series (by its binomial expansion, if $d$ is an integer):

$$
\begin{aligned}
(1-L)^{d} & =\sum_{j=0}^{\infty} \frac{\Gamma(-d+j)}{\Gamma(-d) \Gamma(j+1)} L^{j} \\
& =\sum_{j=0}^{\infty} \frac{(-d)(-d+1) \cdots(-d+j-1)}{j !} L^{j},
\end{aligned}
$$

where

$$
\Gamma(z)=\left\{\begin{array}{cc}
\int_{0}^{\infty} s^{z-1} e^{-z} d s & \text { If } z>0 \\
\infty & z=0 .
\end{array}\right.
$$

If $z<0, \Gamma(z)$ is defined by the recursion formula $z \Gamma(z)=\Gamma(z+1)$.

In recent years, an increasing effort has been made to establish reliable testing procedures to determine whether or not an observed time series is fractionally integrated. In particular, there has been a considerable interest in generalizing the familiar Dickey-Fuller test by taking into account the fractional integration order. It is well documented that the power of Dickey-Fuller $[D F]$ type tests against alternatives of fractional integration is low (see Sowell [23]; Diebold and Rudebusch [9]; Hassler and Wolters [12]; Krämer [14]). This motivated the development of powerful tests against fractional alternatives. Robinson [20] pioneered an integration test constructed from the Lagrange Multiplier $[L M]$ principle, which was proven by Robinson [21] to be locally the most powerful under Gaussianity. The test has been further studied and modified by Agiakloglou and Newbold [1], Tanaka [24]. Tanaka [24] showed, through simulation experiments, that the $L M$ tests have serious size distortion. Another serious criticism addressed to the $L M$ tests is that, by working under the null hypothesis, it does not yield any direct information about the correct long-memory parameter $d$, when the null is rejected (Candelon, Gil Alana [6]).

More recently, Dolado et al [10] introduced a fractional integration test (henceforth $D G M$ test) based on an auxiliary regression for the null of unit $\operatorname{root}\left(H_{0}: d=1\right)$ against the alternative of fractional integration $\left(H_{1}: d=d_{1}\right.$, $\left.d_{1}<1\right)$. Their proposed test reduces to the standard Dickey-Fuller test when $d_{1}=0$ while under the null and when $d_{1}$ known, the statistic in the corresponding regression model depends on a fractional Brownian motion if $0 \leq d_{1}<0.5$. 
Further, the $D G M$ test was refined by Lobato and Velasco ([16], [17]) using the same null and alternative hypotheses.

While the DGM test represents a useful generalization of the Dickey-Fuller test in the presence of a fractionally integrated alternative, it might give arbitrary conclusions when the true $d$ is not present neither in the null nor in the alternative, because the auxiliary regression model, they used, depends on the null and alternative (i.e. 1 and $d_{1}$ ). Indeed, through some simulation experiments we conduct, it may be seen (see Table 1 below) that the $D G M$ test performs somewhat badly in the case where the parameter $d$ is wrongly specified under the null and alternative. In such situation three cases can arise: the case where the null is true, the case where the alternative is true and the case where neither the null nor the alternative is true.

In this paper, we propose an alternative test for the fractional parameter $d$, inspired by the unit root test of Dickey-Fuller [7]. The composite null hypothesis is that of $d \geq d_{0}$ against $d<d_{0}$. The test statistics is the same as in DickeyFuller test using as input $\Delta^{-1+d_{0}} y_{t-1}$ instead of $y_{t-1}$, exploiting the fact that if $y_{t}$ is $I(d)$ then $\Delta^{-1+d_{0}} y_{t}$ is $I(1)$ under the null $d=d_{0}$. If $d \geq d_{0}$, using the generalization of Sowell's result [23], we propose a test based on the least favorable case, $d=d_{0}$, to control type $I$ error, and when $d<d_{0}$ we show that the tests statistics diverges to $-\infty$, providing consistency. Clearly such testing procedure is conceptually attractive since, first, the hypotheses we consider are rather composite-versus-composite $\left(H_{0}: d \geq d_{0}\right.$ against $\left.H_{1}: d<d_{0}\right)$ resulting in a dichotomic choice which excludes the third case. Second, by the choice of a suitable regression model, $\Delta^{d_{0}} y_{t}=\rho \Delta^{-1+d_{0}} y_{t-1}+\varepsilon_{t}$, the usual statistics $t_{\widehat{\rho}_{n}}$ or $n \widehat{\rho}_{n}$ have the same asymptotic distribution as the Dickey-Fuller test. This is because the maximum probability of rejecting the null hypothesis i.e. $\alpha=\operatorname{Sup}_{d \geq d_{0}} P\left(\right.$ reject $\left.H_{0}\right)$, level of the test, is reached when $d=d_{0}$. So, the standard Dickey-Fuller table may be used for our test without an extra-effort i.e. without using the tabulated values of a fractional Brownian motion.

Before going through the topic, it is important to precise certain essential points, which may facilitate the reading of this paper. The main theme of our article is how to extend the familiar Dickey-Fuller [7] type tests for unit root $(I(1)$ against $I(0))$ by embedding the case $d=0$ and $d=1$ in continuum of memory properties (i.e. $d \in \mathbb{R}$ ). Such extension has already been discussed by Dolado et $\mathrm{Al}[10]$. In our paper, we show, in the first step, that the DGM approach is not the best and adequate way to extend the Dickey-Fuller test by taking into account the fractional case. In the second step, we provide how to extend adequately the standard Dickey-Fuller test [7] by taking into account the fractional case.

In order to expose clearly the alternative approach and permit the careful comparison with the $D G M$ approach, we choose to use a simple framework like $\operatorname{ARFIMA}(0, d, 0)(\equiv F I(d))$ process. The case, where the errors are autocorrelated, deserves that one devotes another paper, by taking into account the seminal work of Said and Dickey [22] and Phillips [19]. Our approach is based on the following forth points: 
1 Using the composite hypothesis $H_{0}: d \geq d_{0}$.

2 If $y_{t} \rightsquigarrow I\left(d_{0}\right)$ then $(1-L)^{-1+d_{0}} y_{t} \rightsquigarrow I(1)$.

3 Testing the composite null hypothesis is based upon testing the statistical significance of the coefficient $\phi$ (or $\rho=\phi-1$ ) in the regression model $\Delta^{-1+d_{0}} y_{t}=\phi \Delta^{-1+d_{0}} y_{t-1}+\varepsilon_{t}$.

4 The level of the test $\alpha=\operatorname{Sup}_{d \geq d_{0}} P\left(\right.$ reject $\left.H_{0}\right)=P\left(\right.$ reject $\left.H_{0} \mid d=d_{0}\right)$.

In order to highlight these four important points and not to overlook them into a mid-general framework, the case where the errors are correlated, will not be pursued in this paper. However, I provide (see Appendix 2) some discussions when the process $\left\{y_{t}, t \in \mathbb{Z}\right\}$ is generated by

$$
y_{t}=\mu(t)+F I(d),
$$

with $\mu(t)$ being a vector of deterministic functions like a constant or time trend.

Another reason that led us to choose a simple theoretical framework is to highlight the importance of considering correctly, some basic rules of the testing statistical hypothesis theory. In this paper, we focus on the importance to consider the statistic of the test, exclusively, deduced under the null hypothesis (see section 2, for more details).

The rest of this paper is organized as follows. In section 2 , to highlight the contribution of our approach, we first give some comments on the DGM approach. Then in section 3, we define in a simple framework our test and in particular the auxiliary regression model used to test the null. Moreover, the main results on the asymptotic distribution under the null and alternative composite hypothesis are given. Section 4 explores a theoretical study about the size and power of our proposed $F-D F$ test. Furthermore, Monte-Carlo simulation experiments are undertaken in order to support the analytical results and in particular to confirm that the proposed test is robust to any misspecification of the order of integration parameter $d$. In Section 5 , we present empirical applications by revisiting Nelson-Plosser data. It is important to note that the empirical application is made only to explain how to use the new testing procedure (The reader should not understand this application as to provide a new evidence for the order of integration of the Nelson and Plosser data). Because, as it has been mentioned previously, the data generating process adopted in this paper is restrictive). Finally, the proofs of the main results presented in Section 2 are left to the appendix 1 and some discussions when the process $\left\{y_{t}, t \in \mathbb{Z}\right\}$ is generated by some deterministic trend plus $F I(d)$ process are left to the Appendix 2. 


\section{Fractional Dickey-Fuller testing: the $D G M$ ap- proach}

\subsection{Hypotheses and the auxiliary regression model}

Dolado, Gonzalo, and Mayoral [DGM] [10] introduced a test based on an auxiliary regression for the null of unit root against the alternative of fractional integration. The fractional Dickey-Fuller $(F-D F)$ test considered by $D G M$ [10], in the basic framework, is described as follows.

Let $\left\{y_{t}\right\}_{t=1}^{n}$ a series generated from the fractionally integrated process ( $F I(d)$ in short) given by

$$
(1-L)^{d} y_{t}=u_{t}, t \in \mathbb{Z}
$$

where $d \in \mathbb{R}$ is the true order of integration and, $\left\{u_{t}, t \in \mathbb{Z}\right\}$ is an iid innovation with mean zero and variance $\sigma_{u}^{2}$. For the data generating process $(D G P)(2.1)$, $D G M[10]$ propose to test the following hypotheses,

$$
H_{0}: d=d_{0} \text { against } H_{1}: d=d_{1} \text {, with } d_{1}<d_{0},
$$

by means of the $t$ statistic of the coefficient of $\Delta^{d_{1}} y_{t-1}$ in the ordinary least squares $(O L S)$ regression

$$
\Delta^{d_{0}} y_{t}=\rho \Delta^{d_{1}} y_{t-1}+\varepsilon_{t,}(t=1, \cdots, n)
$$

where $\Delta=1-L . D G M$ explains the choice of the auxiliary regression model (2.3) by arguing that, in the simple Dickey-Fuller test, to test the hypotheses

$$
H_{0}: d=1 \text { against } H_{1}: d=0
$$

the maintained regression model is:

$$
\Delta y_{t}=\rho y_{t-1}+\varepsilon_{t}, \quad t=1, \cdots, n,
$$

where $\varepsilon_{t} \sim i i d\left(0, \sigma_{\varepsilon}^{2}\right)$. If $y_{t}$ is $I(1)$, then the regression (2.5) is unbalanced in the sense that the orders of integration of the regressand and the regressor are different, being $I(0)$ and $I(1)$ respectively. After this, $D G M$ claim that in the simple Dickey-Fuller test the null hypothesis $H_{0}: d=1$ correspond to the regressor $\Delta^{1} y_{t}$ and the alternative $H_{1}: d=0$ correspond to $\Delta^{0} y_{t-1}=y_{t-1}$. This leads them to consider that the null hypothesis $H_{0}: d=d_{0}$ correspond to $\Delta^{d_{0}} y_{t}$ and the alternative $H_{1}: d=d_{1}$ correspond to $\Delta^{d_{1}} y_{t-1}$. In the following we show that the interpretation, made by $D G M$, in the use of the model (2.5), in the simple Dickey-Fuller test is incorrect. In fact, the standard Dickey and Fuller test is not based directly on the regression model (2.5). The hypotheses (2.4) are based on the following regression model

$$
y_{t}=\phi y_{t-1}+\varepsilon_{t} \quad t=1, \cdots, n,
$$


which is equivalent to the regression model (2.5), with $\rho=\phi-1$. The regression model (2.6) is balanced in the sense that the regressand and the regressor have the same order of integration which is equal 1 under the null. The scheme 1 and 2 summarize, respectively, the incorrect and correct interpretation in the use of the model (2.5), in the simple Dickey-Fuller test.

\begin{tabular}{|c|c|}
\hline \multicolumn{2}{|c|}{$\begin{array}{c}\text { Scheme 1: Incorrect interpretation, in the use of model (2.5), } \\
\text { in the simple Dickey-Fuller test }\end{array}$} \\
\hline $\begin{array}{l}D G M \text { interpretation, in the use of the model } \\
(2.5), \text { in the simple Dickey-Fuller test }\end{array}$ & $\begin{array}{l}\text { The use of the incorrect } \\
\text { interpretation in fractional case }\end{array}$ \\
\hline$H_{0}: d=1$ & $H_{0}: d=d_{0} \quad H_{1}: d=d_{1}$ \\
\hline$\Delta \stackrel{\downarrow}{1} y_{t}=\rho \Delta \stackrel{\downarrow}{0}$ & $\Delta \quad \stackrel{\downarrow}{d_{0}} \quad y_{t}=\rho \Delta \quad \stackrel{d_{1}}{y_{t-1}+\varepsilon_{t}}$ \\
\hline
\end{tabular}

\begin{tabular}{|c|c|}
\hline $\begin{array}{r}\text { Scheme 2: Correct interprets } \\
\text { in the } \mathrm{s}\end{array}$ & $\begin{array}{l}\mathrm{n} \text { the use of the models }(2.5) \text { and } \\
\text { Dickey-Fuller test }\end{array}$ \\
\hline $\begin{array}{l}\text { Correct interpretation, in use } \\
\text { of the model }(2.5)\end{array}$ & $\begin{array}{c}\text { Correct interpretation, in the use } \\
\text { of the model }(2.6)\end{array}$ \\
\hline$\overbrace{\Delta y_{t}}^{\text {under } H_{0}}=\rho \overbrace{y_{t-1}}^{\substack{I(1) \\
\text { under }}}+\varepsilon_{t}$ & 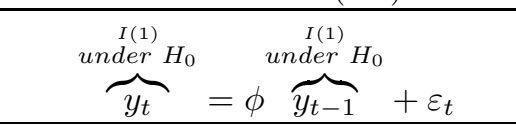 \\
\hline
\end{tabular}

The scheme 2, indicates that the choice of the model (2.5) or equivalently (2.6) is based only on the null hypothesis. The scheme 1, indicates that the incorrect interpretation leads $D G M$ to consider a regression model based on the null and alternative in the fractional case. In fact, for the regression model (2.3) we have, under the null $\left(H_{0}: d=d_{0}\right)$

$$
\Delta^{d_{0}} y_{t} \rightsquigarrow I(0) \quad \text { and } \quad \Delta^{d_{1}} y_{t} \rightsquigarrow I\left(d_{0}-d_{1}\right),
$$

and under the alternative $\left(H_{0}: d=d_{1}\right)$,

$$
\Delta^{d_{0}} y_{t} \rightsquigarrow I\left(d_{1}-d_{0}\right) \quad \text { and } \quad \Delta^{d_{1}} y_{t} \rightsquigarrow I(0) .
$$

As a result, the authors are locked into the trap set by this semblance of analogy. There are other inconsistencies in the use of statistical concepts. Someone, can easily feel, throughout the reading of the article [10], the efforts granted by the authors to justify inconsistencies. It would be long to enumerate all the inconsistencies in the $D G M$ test procedure.

\subsubsection{Unit root test against fractional alternatives and its asymp- totic Properties}

To study the performances of their procedure in terms of power and size, $D G M$

[10] consider only the particular case,

$$
H_{0}: d=1 \text { against } H_{1}: d=d_{1},
$$


by means of the $t$-statistic of the coefficient $\Delta^{d_{1}} y_{t-1}$, in the ordinary least squares $(O L S)$ regression

$$
\Delta^{1} y_{t}=\rho \Delta^{d_{1}} y_{t-1}+\varepsilon_{t} .
$$

The $t$-ratio, $t_{\widehat{\rho}}\left(d_{1}\right)$, is given by

$$
t_{\widehat{\rho}}\left(d_{1}\right)=\frac{\sqrt{n} \sum_{t=2}^{n} \Delta y_{t} \Delta^{d_{1}} y_{t-1}}{\sqrt{\sum_{t=2}^{n}\left(\Delta y_{t}-\widehat{\phi} \Delta^{d_{1}} y_{t-1}\right)^{2} \sum_{t=2}^{n}\left(\Delta^{d_{1}} y_{t-1}\right)^{2}}} .
$$

Theorem 1 (DGM [10]. Under the null hypothesis that $y_{t}$ is a random walk, the asymptotic distribution of $t_{\widehat{\rho}}\left(d_{1}\right)$ is given by

$$
t_{\widehat{\rho}}\left(d_{1}\right) \stackrel{L}{\longrightarrow} \frac{\int w_{-d_{1}}(r) d B(r)}{\left(\int w_{-d_{1}}^{2}(r) d r\right)^{1 / 2}} \text { if } 0 \leq d_{1}<0.5,
$$

and

$$
t_{\widehat{\rho}}\left(d_{1}\right) \stackrel{L}{\longrightarrow} N(0,1) \text { if } 0.5 \leq d_{1}<1 .
$$

where $w_{-d_{1}}(\cdot)$ is fractional Brownian motion.

Proof. See DGM [10]

Theorem 1, shows that under the null the asymptotic distribution of $t$ statistic depends on fractional Brownian motion if $0 \leq d_{1}<0.5$ and $t_{\widehat{\rho}}\left(d_{1}\right) \longrightarrow$ $N(0,1)$ if $0.5 \leq d_{1}<1$. These asymptotic distributions are different from those derived by Dickey and Fuller [7] which depend only on standard Brownian motion. The implementation of $D G M$ [10] test would require tabulation of the percentiles of the functional of Brownian motion, which imply that inference on the presence of unit root would be conditional on $d_{1}$. But given the well-known difficulties in estimating the order of fractional integration in finites samples, thus the test might suffer from misspecification (i.e. the parameter $d$ is wrongly specified)

Under $H_{0}: d=1$, we have $\operatorname{Cov}\left(\Delta^{d_{0}} y_{t}, \Delta^{d_{1}} y_{t-1}\right)=0$ and under the alternative we have $\operatorname{Cov}\left(\Delta^{d_{0}} y_{t}, \Delta^{d_{1}} y_{t-1}\right)=\sigma_{u}^{2}\left(-1+d_{1}\right)<0$. Thus DGM build the decision rule as follows,

$$
\left\{\begin{array}{c}
H_{0}: d=1 \text { is accepted } \quad \text { if } \rho=0 \\
H_{0}: d=d_{1} \text { is accepted } \quad \text { if } \rho<0
\end{array}\right.
$$

The hypotheses (2.7) based on the regression model (2.8) and the decision rule (2.9) is called by their authors "Fractional Dickey and Fuller Test".

Remark 2 Why does DGM consider only the case $H_{0}: d=1$ ? To respond this question, let us consider the case $H_{0}: d=0.5$. Since $\Omega(d)=[0,1]$, the set of alternatives values of $d$ is

$$
\Omega_{1}(d)=[0,1 / 2[\cup] 1 / 2,1] .
$$


In this example, we have two cases. The first case, is given by $d_{0}=1 / 2$ and $d_{1} \in[0,1 / 2[$ and the decision rule is based on " $(\rho=0$ or $\rho<0)$. The second case is given by $d_{0}=1 / 2$ and $\left.\left.d_{1} \in\right] 1 / 2,1\right]$. In this case it is easy to show that the decision rule is based on " $(\rho=0$ or $\rho>0)$ ", because we have

$$
\begin{aligned}
\text { under the null } \operatorname{Cov}\left(\Delta^{0.5} y_{t}, \Delta^{d_{1}} y_{t-1}\right) & =0 \\
\text { under the alternative } \operatorname{Cov}\left(\Delta^{0.5} y_{t}, \Delta^{d_{1}} y_{t-1}\right) & =\sigma_{u}^{2}\left(-0.5+d_{1}\right)>0 .
\end{aligned}
$$

Remark 3 We can suggest another D.G.M. type test. Indeed, since DGM would only use the decision rule (2.9), they would have been better advised if they had thought about testing hypothesis

$$
H_{0}: d=d_{0} \text { against } H_{1}: d=0 \text {, with } d_{0}>0 \text {. }
$$

This choice can be justified by the integration order $d>0$ of the majority of economic series. By using the scheme 1, we can deduce that to test (2.10) we must use the regression model

$$
\Delta^{d_{0}} y_{t}=\rho y_{t-1}+\varepsilon_{t}
$$

With the test hypotheses (2.10) and the regression model (2.11) we can use the decision rule " $(\rho=0$ ou $\rho<0)$, since the case $d<0$ is excluded.

\subsubsection{Power and size of DGM's FDF test.}

The problem with the test based on the hypotheses (2.7) and regression model (2.8) and the test suggested above, based on (2.10) and (2.11) are useless in practice. The problem with the DGM type tests is that they are based on a choice of two possible orders of integration $d_{0}$ and $d_{1}$, of which the true order can be different either in the null or in the alternative. In fact, in the fractional integration case, there is a continuum of possible orders of integration. This would make the simple-versus-simple hypothesis invalid, particularly if the auxiliary regression model, used for the test, is based on the null and alternative. For instance, in the $D G M$ test one of the following three cases holds:

- $d=d_{0}$,

- $d=d_{1}$,

- $d \neq d_{0}$ and $d \neq d_{1}$.

The third case causes serious troubles in practice, particularly, if the statistic of the test depends on null and alternative hypothesis. When $d_{0}=1$, in the first two cases, Dolado et al [10] showed by means of a simulation study that their test procedure has a good performance in terms of power and level. For the third case, Dolado et al [10] studied the effect of hypotheses misspecification by considering the deviations from the true value $d_{1}$ with size $\pm 0.1, \pm 0.2$ and \pm 0.3 . In the following; however, we replicate the simulation results of Dolado 
et al [10] and present them more clearly by using a single table. We generate 1000 series from the data generating process (2.1) with sample size $n=100$. The first column of Table 1 gives the true values of the parameter $d$ while the second line shows the values of $d_{1}$ specified under the alternative. The first line gives the tabulated values by $D G M$ (see Dolado et al [10], table $X$ page 2003). The last line of Table 1 represents the performance of the $D G M$ test in terms of level, i.e. the percentage of rejection of the null, when it is true $(\alpha)$, while the main diagonal represents the performance of the $D G M$ test in terms of power i.e. the percentage of acceptance of the alternative hypothesis when it is true, $(1-\beta)$. $\alpha$ and $\beta$ are respectively the type $I$ and the type $I I$ errors, defined by

$$
\alpha=P\left(\text { reject } H_{0} \mid d=1\right) \quad \text { and } \quad \beta=P\left(\text { reject } H_{1} \mid d=d_{1}\right) \text {. }
$$

The other values in the table are the percentage of acceptance of the alternative hypothesis when both the null and alternative are false i.e. when the value of $d$ is wrongly specified. In fact, these values represents another type of errors, namely

$$
P_{d \neq d_{1}}\left(\text { Accept } H_{1} \mid d \neq 1 \text { and } d \neq d_{1}\right) \text {. }
$$

When performing a test one may arrive at the correct decision, or one may commit one of two errors: rejecting the null hypothesis when it is true (type $I$ error, or error of the first kind) or accepting it when it is false (type $I I$ error or error of the second kind). In statistical testing theory, there is no place for type III error (or error of the third kind). This anomaly is the consequence of the choice of inappropriate auxiliary regression model, which depends on the null and alternative. From Table 1, it may be easily observed that when the true $d$ is well specified, the $D G M$ test has a good performance in terms of power and level. However, in the case where the true value of $d \in[0,1]-\left\{1, d_{1}\right\}$, the conclusions of the test are somewhat arbitrary. For example, when $d=0.3$, the percentage of acceptance of the alternative is equal $100 \%$ regardless of the alternative hypothesis. In other word, if the process $y_{t}$, is fractionally integrated of order $d=0.3$ (i.e. stationary stationary process), the table 1 , show that for $H_{0}: d=1$ against $H_{1}: d=0.7$, we have

$$
P_{d=0.3}\left(\text { Accept } H_{1}: d=0.7 \mid d \neq 1 \text { and } d \neq d_{1}\right)=1 \text {. }
$$

This example shows clearly that the risk to specify the stationary process as a nonstationary process is high.

$L V[17]$ argue that $\Delta^{d_{1}} y_{t-1}$ is not the best class of regression one can choose and propose another auxiliary regression model for the test (2.4). In the case $d_{0}=1$, they propose to test $(2.4)$ by using the following auxiliary model

$$
\Delta y_{t}=\phi_{2} z_{t-1}\left(d_{1}\right)+\varepsilon_{t,}(t=1, \cdots, n),
$$

where

$$
z_{t-1}\left(d_{1}\right)=\left(\frac{\Delta^{d_{1}-1}-1}{1-d_{1}}\right) \Delta y_{t}
$$




\begin{tabular}{|c|c|c|c|c|c|c|c|c|c|c|}
\hline \multicolumn{11}{|c|}{ 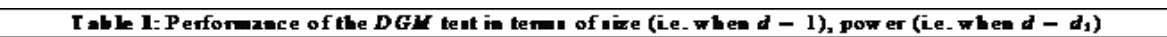 } \\
\hline \multicolumn{11}{|c|}{ and whes the value of $d$ in wrongly upecifued (ie. $d \neq 1$ and $d \neq d_{1}$ ) } \\
\hline $\begin{array}{c}\text { Tabulated } \\
\text { valuex } \\
a-5 \times\end{array}$ & -1.95 & -1.87 & -1.84 & -1.82 & -1.81 & -1.75 & -1.65 & -1.65 & -1.65 & -1.65 \\
\hline True valued $H_{1}=d-d_{1}$ & 0 & 0.1 & 0.2 & 0.9 & 0.4 & 0.5 & 0.6 & 0.7 & 0.8 & 0.9 \\
\hline 0 & 100 & 100 & 100 & 100 & 100 & 100 & 100 & 100 & 100 & 100 \\
\hline 0.1 & 100 & 100 & 100 & 100 & 100 & 100 & 100 & 100 & 100 & 100 \\
\hline 0.2 & 100 & 100 & 100 & 100 & 100 & 100 & 100 & 100 & 100 & 100 \\
\hline 0.9 & 100 & 100 & 100 & 100 & 100 & 100 & 100 & 100 & 100 & 99.9 \\
\hline 0.4 & 100 & 100 & 100 & 100 & 100 & 100 & 100 & 100 & 99.9 & 99.6 \\
\hline 0.5 & 100 & 100 & 100 & 100 & 100 & 100 & 100 & 100 & 100 & 100 \\
\hline 0.6 & 89.5 & 99.5 & 100 & 100 & 100 & 100 & 100 & 100 & 100 & 100 \\
\hline 0.7 & 65.2 & 89.1 & 90.6 & 95.8 & 97.1 & 96.7 & 95.7 & 92.9 & s2. 8 & 82.8 \\
\hline 0.8 & 99.8 & 47.2 & 56.9 & 65.1 & 71.2 & 72.7 & 74.9 & 69.9 & 65.2 & 58.9 \\
\hline 0.9 & 14.7 & 18.4 & 21.2 & 29 & 24.1 & 26.8 & 29.9 & 29 & 27 & 25.2 \\
\hline$H_{0}=d-1$ & 4 & 4_5 & 4.8 & 5.4 & 4_.8 & 4.9 & 5.2 & 5.4 & 5.3 & 5.5 \\
\hline
\end{tabular}

The same criticisms can be formulated concerning test concerning $L V$ test. The $D G M[10]$ and $L V$ [17] tests present an analogy with the original Dickey-Fuller test, but can not be considered as a generalization of the familiar Dickey-Fuller test in the sense that the conventional $I(1)$ vs $I(0)$ framework is recovered (for the $D G M$ test the conventional framework is recovered only if $d_{0}=1$ and $\left.d_{1}=0\right)$. The implementation of $D G M$ [10] test would require tabulations of the percentiles of the functional of fractional Brownian motion, which imply that the inference on the presence of unit root would be conditional on $d_{1}$, and thus might suffer from misspecification resulting from errors in specifying the fractional parameter $d$. When $d_{1}$ is not taken to be known a priory, a preestimation of it is needed to implement the test. In this case, we can perform the test only if the estimator of $d_{1}\left(\widehat{d}_{1}\right)$ is sufficiently close to unity (see $D G M$ [10] for more details). Indeed, the table 1, show that the $D G M$ test have "a realistic" behavior (i.e. likely results) in terms of size and power only when the true value of $d$ is close to 1 (see for instance in table $1:, d=0.8$ and $d=0.9$ ). This is why, DGM recommend to use an estimator of $d_{1}$ that originates from the trimming rule

$$
\widehat{d}_{1}=\left\{\begin{array}{c}
\widehat{d}_{n}, \quad \text { if } \widehat{d}_{n}<1-c \\
1-c \quad \text { if } \widehat{d}_{n} \geq 1-c
\end{array} \quad, 0<c<0.5,\right.
$$

where $\widehat{d}_{n}$ is any $\sqrt{n}$-consistent estimator of $d_{1}$, for example $D G M$ select $c=0.02$ in their simulation experiments. This rule makes this test more vague in how to use it in practice.

To extend adequately the standard Dickey-Fuller test [7], we propose a new test based on mutually exclusive and complementary null, alternative hypotheses and a suitable auxiliary regression model. 


\section{Fractional Dickey-Fuller testing: an alterna- tive approach}

\subsection{Hypotheses, the auxiliary regression model and asymp- totic under the null and the alternative}

In this section, we deal with a series $\left\{y_{t}\right\}_{t=1}^{n}$ generated from the fractionally integrated model, $F I(d)$, given by (2.1), where the order $d$ is any real number. Under this setting, we propose to test the following hypotheses :

$$
H_{0}: d \geq d_{0} \quad \text { against } \quad H_{1}: d<d_{0} .
$$

Our proposal is based upon testing the statistical significance of the coefficient $\phi($ or $\rho=\phi-1)$ in the following regression model,

$$
\Delta^{-1+d_{0}} y_{t}=\phi \Delta^{-1+d_{0}} y_{t-1}+\varepsilon_{t},
$$

or equivalently

$$
\Delta^{d_{0}} y_{t}=\rho \Delta^{-1+d_{0}} y_{t-1}+\varepsilon_{t}
$$

where $\rho=\phi-1$ and $\left\{\varepsilon_{t}\right\}_{t=1}^{n}$ are the residuals. The most important idea behind the choice of the framework above is that

$$
\text { when } d=d_{0}, \quad x_{t}=\Delta^{-1+d_{0}} y_{t} \text {, is integrated of order } 1 \text {. }
$$

More generally,

$$
x_{t} \text { is integrated of order } 1+d-d_{0},
$$

with

$$
\left\{\begin{array}{l}
1+d-d_{0} \geq 1, \text { if } d \geq d_{0}, \\
1+d-d_{0}<1, \text { if } d<d_{0} .
\end{array}\right.
$$

Before stating the main results of this paper, we give some technical tools that we need in the sequel. Let $\eta_{t}=(1-L)^{-\delta} u_{t}$, with $\left.\left.\delta \in\right]-0.5,0.5\right]$ and $u_{t}$ defined as above. Let $\sigma_{S}^{2}=\operatorname{var}\left(S_{n}\right)$, where $S_{t}=\sum_{j=1}^{t} \eta_{j}$. When $|\delta|<\frac{1}{2}$, we have (see Sowell [23])

$$
\lim _{n \rightarrow \infty} n^{-1-2 \delta} \sigma_{S}^{2}=\frac{\sigma_{\varepsilon}^{2} \Gamma(1-2 \delta)}{(1+2 \delta) \Gamma(1+\delta) \Gamma(1-\delta)} \equiv \kappa_{\eta}^{2}(\delta),
$$

where $\Gamma(\cdot)$ denotes the Gamma or generalized factorial function. For the case $\delta=\frac{1}{2}$, (see Liu, [15])

$$
\lim _{n \rightarrow \infty}\left(n^{-2} \log ^{-1} n\right) \sigma_{S}^{2}=\frac{2 \sigma_{\varepsilon}^{2}}{\pi} \equiv \kappa_{\eta}^{2}\left(\frac{1}{2}\right) .
$$

\footnotetext{
${ }^{3}$ The special case of hypothesis testing $H_{0}: d \geq 1$ against $H_{1}: d<1$ was presented at ICMSAO'13 Conference, Hammamet, Tunisia, 28-30 April 2013, in the paper entitled "A consistent test for unit root against fractional alternative". Expanded version of this paper forthcoming in Inderscience journal "International Journal of operational research "

${ }^{4}$ This paper is an expanded version of the paper entitled "New fractional Dickey-Fuller test" presented at ICMSAO'15 conference, Istanbul, May 27-29,2015
} 
Furthermore, under the following additional assumption $E\left|u_{t}\right|^{a}<\infty$, for some $a \geq \max \left\{4, \frac{-8 \delta}{1+2 \delta}\right\}$, the following useful results apply:

$$
n^{-\frac{1}{2}-\delta} \kappa_{\eta}^{-1}(\delta) S_{[n r]} \Rightarrow \frac{1}{\Gamma(1+\delta)} \int_{0}^{r}(r-s)^{\delta} d \mathbf{w}(s), \quad \text { when }-\frac{1}{2}<\delta<\frac{1}{2}
$$

and

$$
n^{-\frac{1}{2}-\delta}\left(\log ^{-1} n\right) \kappa_{\eta}^{-1}\left(\frac{1}{2}\right) S_{[n r]} \Rightarrow \mathbf{w}_{0.5}(r), \quad \text { when } \delta=0.5,
$$

where $\mathbf{w}(r)$ is the standard Brownian motion on $[0,1]$ associated with the $\left(u_{t}\right)$ sequence and the symbols $" \Rightarrow$ " and " $\stackrel{p}{\rightarrow}$ " denotes respectively weak convergence and convergence in probability.

Since $d-d_{0}$ can always be decomposed as $d-d_{0}=m+\delta$, where $m \in \mathbb{N}$ and $\delta \in]-0.5,0.5]$, the following result provides the asymptotic distribution of the Dickey-Fuller normalized bias statistic $n \widehat{\rho}_{n}=n\left(\widehat{\phi}_{n}-1\right)$ and the Dickey-Fuller t-statistic, $t_{\widehat{\rho}_{n}}$, in the least square esimate of the model (3.3).

Theorem 4 Let $\left\{y_{t}\right\}_{t=1}^{n}$ be generated from the DGP (2.1). If the regression model (3.3) is fitted to a sample of size $n$ then, as $n \uparrow \infty$,

1. $n \widehat{\rho}_{n}$ satisfies

$$
\begin{array}{r}
\widehat{\rho}_{n}=O_{p}(1) \text { and } n \widehat{\rho}_{n} \stackrel{p}{\rightarrow}-\infty, \text { if }-1 \leq d-d_{0}<-0.5, \\
\widehat{\rho}_{n}=O_{p}\left(\log ^{-1} n\right) \text { and } n \widehat{\rho}_{n} \stackrel{p}{\rightarrow}-\infty, \text { if } d-d_{0}=-0.5, \\
\widehat{\rho}_{n}=O_{p}\left(n^{-1-2 \delta}\right) \text { and } n \widehat{\rho}_{n} \stackrel{p}{\rightarrow}-\infty, \text { if }-0.5<d-d_{0}<0, \\
\widehat{\rho}_{n}=O_{p}\left(n^{-1}\right) \text { and } n \widehat{\rho}_{n} \Rightarrow \frac{\frac{1}{2}\left\{\mathbf{w}^{2}(1)-1\right\}}{\int_{0}^{1} \mathbf{w}^{2}(r) d r}, \text { if } d-d_{0}=0, \\
\widehat{\rho}_{n}=O_{p}\left(n^{-1}\right) \text { and } n \widehat{\rho}_{n} \Rightarrow \frac{\frac{1}{2} \mathbf{w}_{\delta, m+1}^{2}(1)}{\int_{0}^{1} \mathbf{w}_{\delta, m+1}^{2}(r) d r}, \text { if } d-d_{0}>0 .
\end{array}
$$

2. $t_{\widehat{\rho}_{n}}$ is such that

$$
\begin{gathered}
t_{\widehat{\rho}_{n}}=O_{p}\left(n^{0.5}\right) \text { and } t_{\widehat{\rho}_{n}} \stackrel{p}{\rightarrow}-\infty, \text { if }-1 \leq d-d_{0}<-0.5, \\
t_{\widehat{\rho}_{n}}=O_{p}\left(n^{0.5} \log ^{0.5} n\right) \text { and } t_{\widehat{\rho}_{n}} \stackrel{p}{\rightarrow}-\infty \text {, if } d-d_{0}=-0.5, \\
t_{\widehat{\rho}_{n}}=O_{p}\left(n^{-\delta}\right) \text { and } t_{\widehat{\rho}_{n}} \stackrel{p}{\rightarrow}-\infty, \text { if }-\frac{1}{2}<d-d_{0}<0, \\
t_{\widehat{\rho}_{n}}=O_{p}(1) \text { and } t_{\widehat{\rho}_{n}} \Rightarrow \frac{\frac{1}{2}\left\{\mathbf{w}^{2}(1)-1\right\}}{\left[\int_{0}^{1} \mathbf{w}^{2}(r) d r\right]^{1 / 2}} \text {, if } d-d_{0}=0, \\
t_{\widehat{\rho}_{n}}=O_{p}\left(n^{\delta}\right) \text { and } t_{\widehat{\rho}_{n}} \stackrel{p}{\rightarrow}+\infty, \text { if } 0<d-d_{0}<0.5,
\end{gathered}
$$




$$
t_{\widehat{\rho}_{n}}=O_{p}\left(n^{0.5}\right) \text { and } t_{\widehat{\rho}_{n}} \stackrel{p}{\rightarrow}+\infty, \text { if } d-d_{0} \geq 0.5 .
$$

where $\mathbf{w}_{\delta, m}(r)$ is the $(m-1)$-fold integral of $\mathbf{w}_{\delta}(r)$ recursively defined as $\mathbf{w}_{\delta, m}(r)=\int_{0}^{r} \mathbf{w}_{\delta, m-1}(s) d s$, with $\mathbf{w}_{\delta, 1}(r)=\mathbf{w}_{\delta}(r)$ and $\mathbf{w}(r)$ denotes the standard Brownian motion.

Proof. See Appendix.

The later properties represent generalizations of those established by Sowell [23] for the cases $-\frac{1}{2}<d-1<0, d-1=0$ and $0<d-1<\frac{1}{2}$. From (3.8), (3.9) and (3.10), the rate at which $\widehat{\rho}_{n}=\widehat{\phi}_{n}-1$ converges to zero (i.e. $\widehat{\phi}_{n}$ converge to 1 ) is slow for non-positive values of $d-d_{0}$, and is particularly very slow for $-\frac{1}{2}<d-d_{0}<-\frac{1}{4}$. Moreover for $-\frac{1}{2}<d-d_{0}<0$, the limiting distribution of $\widehat{\rho}_{n}$ has non-positive support and then $\lim _{n \rightarrow \infty} P\left(\widehat{\phi}_{n}<1\right)=1$. From (3.11) and (3.12), $\widehat{\rho}_{n}$ converges to zero at rate $n$, when $d \geq d_{0}$. The rate of convergence $n$ is faster than the usual standard rate $n^{\frac{1}{2}}$, when we deal with stationary $I(0)$ variables. Then, for $d-d_{0} \geq 0$, the least squares estimate is superconsistent. In other words, if a first order autoregression (3.2) is fitted to a series generated from an ARFIM $A\left(0,1+d-d_{0}, 0\right)$, where $1+d-d_{0}$ is the order of integration of $\Delta^{-1+d_{0}} y_{t}$, then when $d-d_{0} \geq 0$, asymptotically, the $O L S$ estimator $\widehat{\phi}_{n}$ will not exceed 1 in probability. Figure 1 and Figure 2 below illustrate this fact in an obvious way.

Figure 1: Relation between the order of integration, $d$, of the process $y_{t}$ and the OLS estimator, $\widehat{\phi}_{n}$, in the regression model $\Delta^{-1+d_{0}} y_{t}=\phi \Delta^{-1+d_{0}} y_{t-1}+\varepsilon_{t},\left(d_{0}\right.$ fixed and $d$ varied)

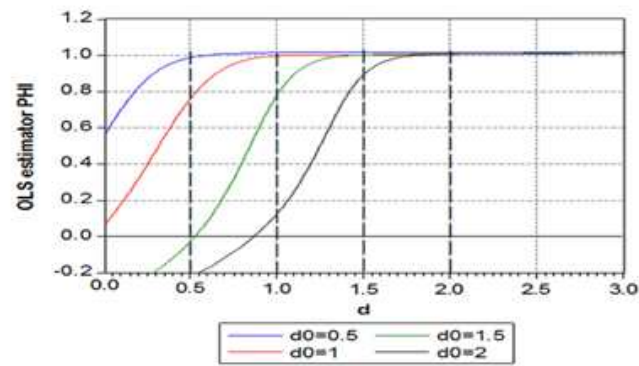

Figure 1 shows that $\widehat{\phi}_{n}=1$ as long as $d-d_{0} \geq 0$, and $\widehat{\phi}_{n}<1$ as long as $d-d_{0}<0$, where $\widehat{\phi}_{n}$ is the $O L S$ estimator in the autoregression model (3.2).

Example 5 For example, for $d_{0}=0.5$, we have,

$$
\left\{\begin{array}{c}
d-0.5<0 \text { and } \widehat{\phi}_{n}<1 \text { for } 0 \leq d<0.5 \\
d-0.5 \geq 0 \text { and } \widehat{\phi}_{n}=1 \text { for } d \geq 0.5
\end{array}\right.
$$


and for $d_{0}=2$, we have,

$$
\left\{\begin{array}{c}
d-2<0 \text { and } \widehat{\phi}_{n}<1 \text { for } 0 \leq d<2 \\
d-2 \geq 0 \text { and } \widehat{\phi}_{n}=1 \text { for } d \geq 2
\end{array}\right.
$$

Figure 2: Relation between the order of integration, $d$, of the process $y_{t}$ and the $O L S$ estimator, $\widehat{\phi}_{n}$, in the regression model $\Delta^{-1+d_{0}} y_{t}=\phi \Delta^{-1+d_{0}} y_{t-1}+\varepsilon_{t},(d$ fixed and $d_{0}$ varied)

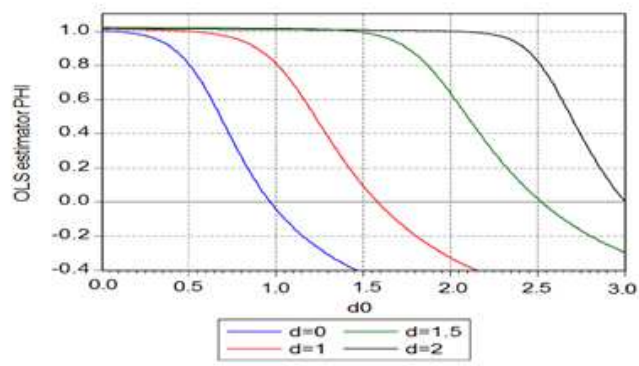

Figure 2 shows that as long as $d-d_{0} \geq 0$, we have $\widehat{\phi}_{n}=1$, and $\widehat{\phi}<1$ whenever $d-d_{0}<0$, where $\hat{\phi}_{n}$ is the $O L S$ estimator in the autoregression model (3.2).

Example 6 For example, when $d=0.5$,

$$
\left\{\begin{array}{c}
0.5-d_{0}<0 \text { and } \widehat{\phi}_{n}<1 \text { for } 0 \leq d_{0}<0.5, \\
0.5-d_{0} \geq 0 \text { and } \widehat{\phi}_{n}=1 \text { for } d_{0} \geq 0.5,
\end{array}\right.
$$

and when $d=2$,

$$
\left\{\begin{array}{c}
2-d_{0}<0 \text { and } \widehat{\phi}_{n}<1 \text { for } 0 \leq d_{0}<2, \\
2-d_{0} \geq 0 \text { and } \widehat{\phi}_{n}=1 \text { for } d_{0} \geq 2
\end{array}\right.
$$

Figure 1 is made as follows: For a fixed sample $\left\{u_{0}, \cdots, u_{1000}\right\}$ generated from a Gaussian i.i.d. $(0,1)$ process, samples of $A R F I M A\left(0,1+d-d_{0}, 0\right)$ processes were generated for $d$ varying between 0 and 3 with step-size 0.01 and $d_{0}$ fixed. Similarly, Figure 2 is made as follows. For a series $\left\{u_{0}, \cdots, u_{1000}\right\}$ generated from a Gaussian i.i.d. $(0,1)$ process, samples from $\operatorname{ARFIMA}\left(0,1+d-d_{0}, 0\right)$ processes were generated for $d_{0}$ varying between 0 and 3 with step-size 0.01 for fixed $d$. For each series $\left\{x_{t}, t=1, \cdots, 1000\right\}$, a first order autoregression (3.2) is fitted and an estimate of $\phi$ is calculated. By plotting the estimate $\widehat{\phi}_{n}$ against the fractional parameter $d$, one obtains Figure 1 and by plotting the parameter $\widehat{\phi}_{n}$ against the fractional parameter $d_{0}$ one obtains Figure 2 . A general procedure for generating a fractionally integrated series with length $n$ is to apply the formula $x_{t}=\sum_{j=0}^{t-1} \frac{\Gamma\left(d+1-d_{0}+j\right)}{\Gamma\left(d+1-d_{0}\right) \Gamma(j+1)} u_{t-j}$ for $t=1, \ldots, n$. 


\section{Remark 7}

By fixing the parameter $d_{0}$ and varying the parameter $d$, we increase the order of integration of $x_{t}$, and by varying the parameter $d_{0}$ and fixing the parameter $d$ we decrease the order of integration of $x_{t}$.

The relationships between $\widehat{\phi}_{n}$ and $d$ and between $\widehat{\phi}_{n}$ and $d_{0}$, highlighted by the results (3.8)-(3.12) and illustrated by Figures 1 and 2, suggest that when we deal with testing the degree of fractional integration, we have

$$
H_{0}: d \geq d_{0} \Rightarrow H_{0}: \phi=1 \text { and } H_{1}: d<d_{0} \Rightarrow H_{1}: \phi<1 .
$$

Like $D G M$, we call the test which is based on the hypotheses (3.1) and the auxiliary regression model (3.2), or equivalently (3.3) as "Fractional DickeyFuller test ( $F-D F$ test in short).

Another important property highlighted by Theorem 3.1 is that the tests are invariant to the original value of $d$, so the asymptotic properties only depend on $d-d_{0}$. For example, we have used several series with sample size 10000 to estimate the densities (following Sowell [23]) of $n \widehat{\rho}_{n}$ and $t_{\widehat{\rho}_{n}}$ under $d-d_{0}=0$. The estimated densities are presented in Figures 3 and 4 above.

Figure 3: Kernel density estimate of $n \widehat{\rho}$ statistic under $H_{0}: d=d_{0}$ using 1000 samples of size $n=250 . \quad\left(n \widehat{\rho}_{n} \Rightarrow \frac{\frac{1}{2}\left\{\mathbf{w}^{2}(1)-1\right\}}{\int_{0}^{1} \mathbf{w}^{2}(r) d r}\right)$

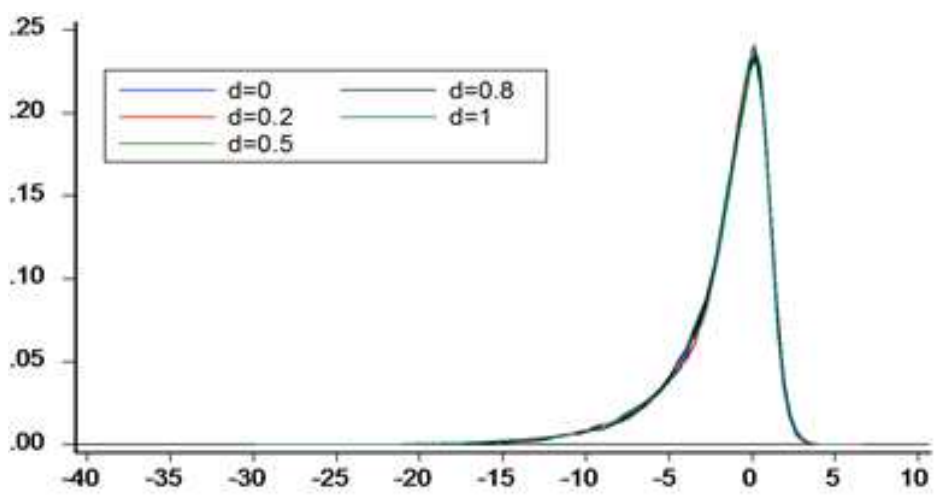

In figures 3 and 4 , for each one of the statistics $n \widehat{\rho}_{n}$ and $t_{\widehat{\rho}_{n}}$ under $d=d_{0}$ with $n=250$, the estimated densities for different values of $d$ are represented on the same graph. Figures 3 and 4 show that by fitting the regression model (3.3) to the sample generated from (2.1), one obtains the same distribution as those used by Dickey-Fuller [7]. In other words, as shown below, the proposed test, which is based on the regression model (3.2) (or equivalently (3.3)) and 
Figure 4: Kernel density estimate of $t_{\widehat{\rho}}$ statistic under $H_{0}: d=d_{0}$ using 1000 samples of size $n=250 . \quad\left(t_{\widehat{\rho}_{n}} \Rightarrow \frac{\frac{1}{2}\left\{\mathbf{w}^{2}(1)-1\right\}}{\left[\int_{0}^{1} \mathbf{w}^{2}(r) d r\right]^{1 / 2}}\right)$

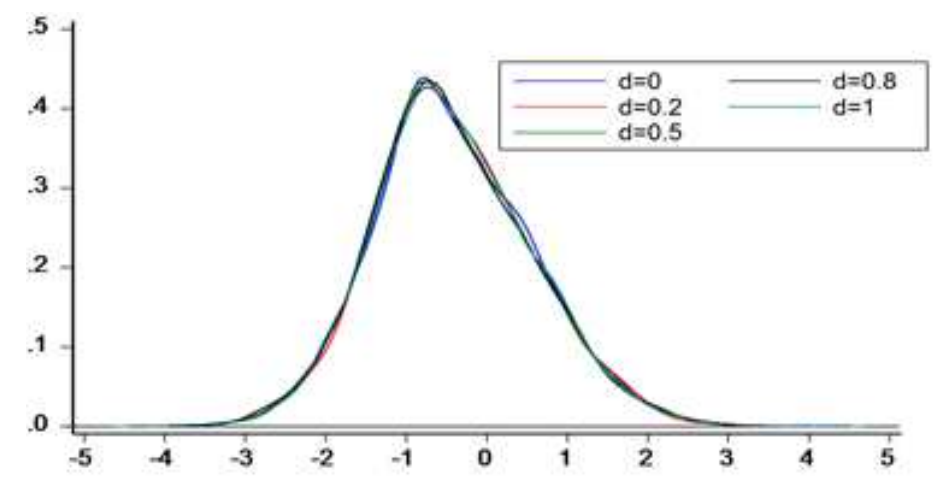

the composite hypotheses (3.1), can be understood and implemented exactly as the simple Dickey-Fuller test for unit root by using the usual statistical tables of the conventional statistics $n \widehat{\rho}_{n}$ and $t_{\widehat{\rho}_{n}}$.

\section{Power and size of the $F-D F$ test}

\subsection{Some theoretical aspects}

Let $Z_{1}=n\left(\widehat{\phi}_{n}-1\right)=n \widehat{\rho}_{n} \quad$ and $\quad Z_{2}=t_{\widehat{\rho}_{n}}$. For a composite hypothesis, the parameter space $\Omega=\mathbb{R}$ is divided into disjoint regions, $\Omega_{0}=\left[d_{0},+\infty[\right.$ and $\left.\Omega_{1}=\right]-\infty, d_{0}[$. The test is written

$$
H_{0}: d \in \Omega_{0} \quad \text { against } \quad H_{1}: d \in \Omega_{1} \text {. }
$$

For a series generated from (2.1) with sample size $n$ we introduce two nonrandomized test defined by a function $\boldsymbol{\Psi}_{i, n}, i=1,2$ on the sample space of the observations $Z_{i}, i=1,2$ with critical regions $C_{i}, i=1,2$. The test $\boldsymbol{\Psi}_{i, n}$ for a given rejection region $C_{i}$ is

$$
\Psi_{i, n}\left(z_{i}\right)=\left\{\begin{array}{l}
1 \text { if } z_{i} \in C_{i}, \\
0 \text { if } z_{i} \notin C_{i} .
\end{array}\right.
$$

The power of the test is defined by the function

$$
\Pi_{\Psi_{i, n}}(d)=P\left[Z_{i} \in C_{i} \mid d\right] .
$$


$\Pi_{\Psi_{i, n}}(d)$ measures the probability of rejecting the null hypothesis for a given $d$ and rejection region $C_{i}$. The ideal test function has

$$
\Pi_{\Psi_{i, n}}(d) \approx 0 \text { for all } d \in \Omega_{0} \text { and } \quad \Pi_{\boldsymbol{\Psi}_{i, n}}(d) \approx 1 \text { for all } d \in \Omega_{1},
$$

and the test function yields the correct decision with probability nearly 1 . The type $I$ and type $I I$ errors can be summarized in the power function $\Pi_{\Psi_{i, n}}(d)$. For $d \in \Omega_{0}$,

$\Pi_{\Psi_{i, n}}(d)$ is the probability of making a type $I$ error (size of the test), and for $d \in \Omega_{1}$,

$1-\Pi_{\Psi_{i, n}}(d)$ is the probability of making a type $I I$ error.

For the alternative hypothesis $H_{1}: d<d_{0}$, we consider the one sided critical regions of the form

$$
C_{i}=\left\{Z_{i}<c_{n, i}(\alpha)\right\},
$$

where $\alpha$ is the level of the test and $c_{n, i}(\alpha)$ the critical points. The level $\alpha$ of the test $\boldsymbol{\Psi}_{i, n}$ is given by

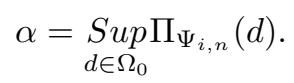

It measures the maximum probability of rejecting the null hypothesis when it is true. For the statistic $Z_{1}$, the figures 5 and 6 , where $c=c_{n, 1}(\alpha)$, show clearly that the supremum occurs at $d=d_{0}$,

$$
P\left[Z_{1} \in C_{1} \mid d \in \Omega_{0}\right] \leq P\left[Z_{1} \in C_{1} \mid d=d_{0}\right] .
$$

For the statistic $Z_{2}$, the figure 7 shows also clearly that

$$
P\left[Z_{2} \in C_{2} \mid d \in \Omega_{0}\right] \leq P\left[Z_{2} \in C_{2} \mid d=d_{0}\right],
$$

because the asymptotic distribution of $Z_{2}$ is well defined, in all the real line for $d=d_{0}$ and diverge to $+\infty$ for $d>d_{0}$. The figures 5,6 and 7 show that the level of the test is

$$
\alpha=\underset{d \in \Omega_{0}}{\operatorname{Sup} \Pi_{\Psi_{i, n}}}(d)=\Pi_{\Psi_{i, n}}\left(d_{0}\right) .
$$

Another technical argument that supports (4.4), is that the asymptotic distributions of $Z_{1}$ and $n^{-\delta} Z_{2}, \delta>0$, have positive support for $d>d_{0}$ and well defined in all the real line for $d=d_{0}$. Consequently, the critical points $c_{n, i}(\alpha), i=1,2$ are those used in the simple Dickey-Fuller test (i.e. without trend and intercept). Note that all the critical values $c_{n, i}(\alpha)$ are less than zero for $\alpha=1 \%$, $5 \%$ and $1 \%$. As indicated by (4.4), a test has level $\alpha$ if its size is less than $\alpha$. 
Figure 5: Kernel density estimates of $n \hat{p}_{n}$ using 10000 samples of size $n=250$, when the values of $d$ are respectively $1,1.2,1.3,1.4$, and the value specified under the null is $d_{0}=1$

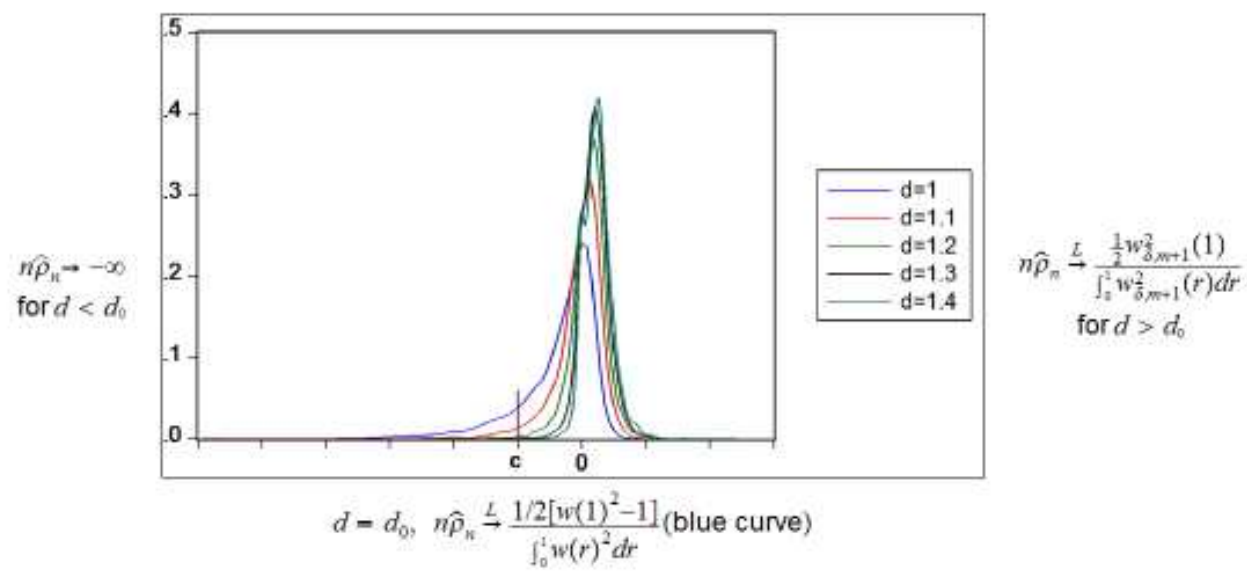

Figure 6: Kernel density estimates of $n \hat{p} \hat{\nu}_{n}$ using 10000 samples of size $n=250$, when the values of $\mathrm{d}$ are respectively $0.5,0.6,0.7,0.8,0.9,1.5$ and the value specified under the null is $d_{0}=0.5$

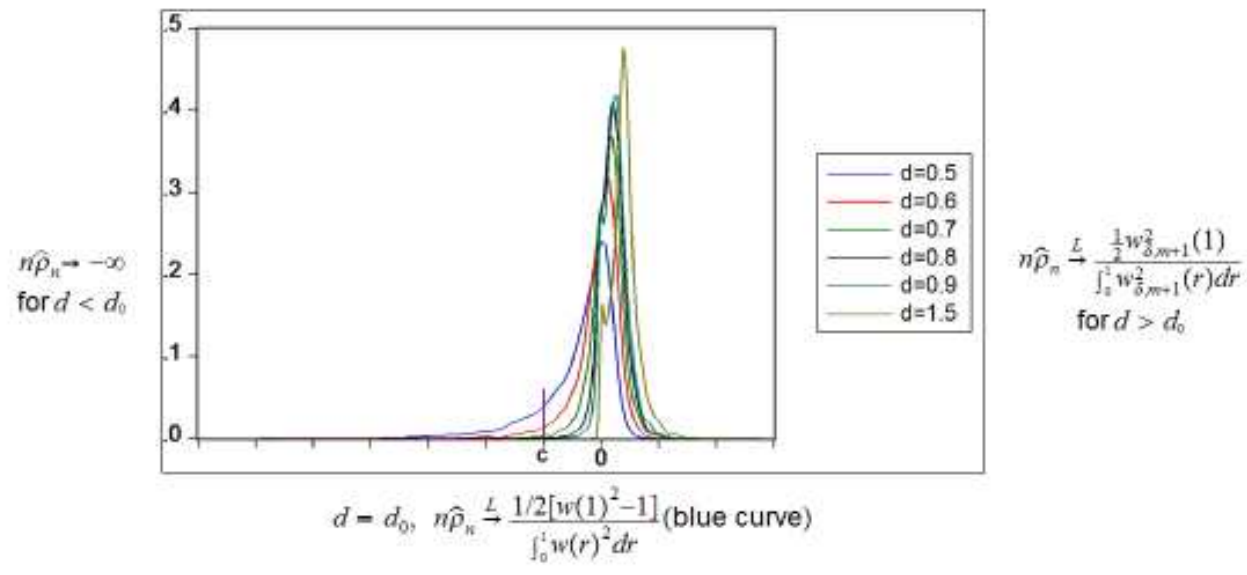


Figure 7: Asumptotic under the null and alternative of the statistic $Z_{2}$ and the rejection region (red colour)

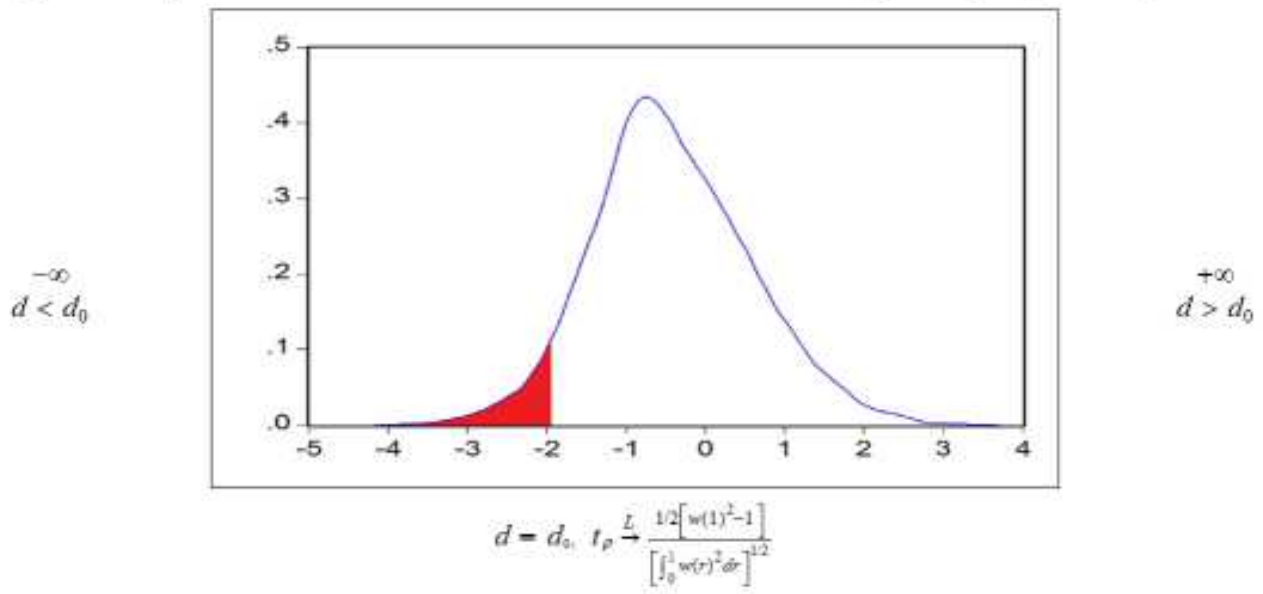

Theorem 8 For a given level $\alpha$, a sequence of test $\left\{\Psi_{i, n}\right\}, i=1,2$, defined by (4.1), with critical region (4.2) is consistent i.e.

$$
\begin{aligned}
& \operatorname{Lim}_{n \rightarrow \infty} \Pi_{\Psi_{i, n}}(d)=0, \text { for } d-d_{0} \geq 0, \\
& \operatorname{Lim}_{n \rightarrow \infty} \Pi_{\Psi_{i, n}}(d)=0, \text { for }-0.5<d-d_{0}<0 .
\end{aligned}
$$

Proof. First, we consider the statistic $Z_{1}$. For $-0.5<d-d_{0}<0$ (i.e. $-0.5<$ $\delta<0)$, since from result, $(3.10)$, the asymptotic distribution of $n^{2 \delta} Z_{1}$ has nonpositive support and $\underset{n \rightarrow \infty}{\operatorname{Lim}} n^{2 \delta} c_{n, 1}(\alpha)=0$, then we have

$$
\begin{aligned}
\operatorname{Lim}_{n \rightarrow \infty} P\left[Z_{1}<c_{n, 1}(\alpha) \mid d<d_{0}\right] & =\operatorname{Lim}_{n \rightarrow \infty} P\left[n^{2 \delta} Z_{1}<n^{2 \delta} c_{n, 1}(\alpha)\right], \\
& =1 .
\end{aligned}
$$

We have, also, $\operatorname{Lim}_{n \rightarrow \infty} P\left[Z_{1}<c_{n, 1}(\alpha) \mid d>d_{0}\right]=0$, because $Z_{1}$ has a positive support for $d>d_{0}, c_{n, 1}(\alpha)<0$ and $\operatorname{Lim}_{n \rightarrow \infty} n^{2 \delta} c_{n, 1}(\alpha)=0$. Now consider the statistic $Z_{2}$. For For $-0.5<d-d_{0}<0$ (i.e. $-0.5<\delta<0$ ), using the same arguments as above we have,

$$
\begin{aligned}
\operatorname{Lim}_{n \rightarrow \infty} P\left[Z_{2}<c_{n, 2}(\alpha) \mid d<d_{0}\right] & =\operatorname{Lim}_{n \rightarrow \infty} P\left[n^{\delta} Z_{2}<n^{\delta} c_{n, 2}(\alpha)\right], \\
& =1 .
\end{aligned}
$$

For $d>d_{0}$, with $\gamma=\delta$, when $0<\delta=d-d_{0}<0.5$ and $\gamma=0.5$ when $d-d_{0} \geq 0.5$, we have

$$
\operatorname{Lim}_{n \rightarrow \infty} P\left[n^{-\gamma} Z_{2}<n^{-\gamma} c_{2, n}(\alpha) \mid d>d_{0}\right]=0 .
$$




\subsection{Simulation study}

In this subsection, through a Monte Carlo study, we show that the proposed $F D-F$ test performs very well in terms of power and size when we use the $t$ statistic. To investigate the size and power of the $F$-DF test, 10000 samples of $F I(d)$ Gaussian processes (2.1) are generated and the regression model (3.3) is used to estimate $t$. The sample-sizes considered are $n=50$ and $n=250$. Three values of $d$ are used: $0 ; 0.5 ; 1$. For each value, we specify the various values for $d_{0}$. Letting $S_{d}\left(d_{0}\right)$ be the set of values of $d_{0}$ for a given value of $d$, the sets which will be used for the three values of $d$ are respectively

$$
\begin{aligned}
S_{0}\left(d_{0}\right) & =\{-0.4 ;-0.3 ;-0.2 ;-0.1 ; \mathbf{0} ; 0.1 ; 0.2 ; 0.3 ; 0.4\}, \\
S_{0.5}\left(d_{0}\right) & =\{0 ; 0.1 ; 0.2 ; 0.3 ; 0.4 ; \mathbf{0 . 5} ; 0.6 ; 0.7 ; 0.8 ; 0.9\}, \\
S_{1}\left(d_{0}\right) & =\{0.5 ; 0.6 ; 0.7 ; 0.8 ; 0.9 ; \mathbf{1} ; 1.1 ; 1.2 ; 1.3 ; 1.4\} .
\end{aligned}
$$

Table 2 and Table 3 give simulation results on the size of the test, (i.e. when $d-d_{0}=\delta \geq 0$ ), where it may be easily seen that the $F-D F$ test, based on the auxiliary regression model (3.3), has good performances in terms of size since

$$
P\left(t<c_{n}(\alpha) \mid \delta>0\right) \leq P\left(t<c_{n}(\alpha) \mid \delta=0\right) \text { and } P\left(t<c_{n}(\alpha) \mid \delta=0\right) \approx \alpha .
$$

Table 2 and Table 3 provide, also, the simulation results on the power of the test (i.e. when $d-d_{0}=\delta<0$ )

$$
P\left(t<c_{n}(\alpha) \mid \delta<0\right) \geq P\left(t<c_{n}(\alpha) \mid \delta=0\right) .
$$

In this case, there are some conclusions to be drawn from it. First, the power of the $F-D F$ test increases with the increase of sample size and $\delta=d-d_{0}$. For example, for $\alpha=5 \%, d=1$ and $\delta=-0.1$, the power is $12.36 \%$ for $n=50$ and $20.76 \%$ for $n=250$. When $\alpha=5 \%, d=1$ and $\delta=-0.3$, the power is $48.5 \%$ for $n=50$ and $86.05 \%$ for $n=250$. Second, as shown in table 3, for $n=250$, the power of the $F-D F$ test is below $50 \%$ for $(\delta=-0.1)$ and for $(\alpha=1 \%$, $\delta=-0.2$ ). Third, for given $n, \alpha$ and $\delta$, the power for $d=0, d=0.5$ and $d=1$ are approximately similar because the asymptotic under the alternative does not depend on $d$ but only on $\delta=d-d_{0}$. Finally, another important property showed by the table 2 and 3 is that the power function satisfies

$$
\begin{array}{ll}
\Pi_{\Psi_{i, n}}(d) \leq \alpha & \text { if } d-d_{0} \geq 0 \\
\Pi_{\Psi_{i, n}}(d) \geq \alpha & \text { if } d-d_{0}<0 .
\end{array}
$$

A test for which the power function satisfies the conditions above is said to be unbiased.

Similar results are obtained for the $n \widehat{\rho}_{n}$ statistic. Since $n \widehat{\rho}_{n}$ has the non degenerate limit distribution, we choose to give the simulation results in the form of estimation density (by kernel methods, like Sowell [23]. The figures 5 and 6 above summarize these results and support clearly those of Theorem 4.1. 


\begin{tabular}{|c|c|c|c|c|c|c|c|c|c|c|}
\hline True vakis of $d$ & $\sigma \backslash \bar{\sigma}$ & 0.4 & 0.3 & 0.2 & 0.1 & 0 & -0.1 & -0.2 & -0.3 & -0.4 \\
\hline \multirow{3}{*}{$d=0$} & $1 \%$ & 0.00 & 0.01 & 0.07 & 0.27 & 1.04 & 3.61 & 9.53 & 2223 & 45.82 \\
\hline & $5 \%$ & 0.02 & 0.15 & Q.61 & 1.83 & 5.95 & 1266 & 26.68 & 47.16 & 73.03 \\
\hline & $10 \% \mathrm{~b}$ & Q.15 & 0.52 & 1.74 & 4.22 & 9.66 & 21.97 & 39,56 & 6.14 & 84.92 \\
\hline \multirow{3}{*}{$d=0.5$} & $1 \%$ & 0.02 & 0.03 & Q.1 & 0.26 & 1.09 & 2.87 & 9.36 & 22.59 & 45.16 \\
\hline & 15: & 0.09 & 0.22 & e. 72 & 1.86 & 4.78 & 1190. & 24.17 & 48.34 & 72.74 \\
\hline & $10 \%$ & 0.22 & 0.58 & 1.75 & 4.37 & 10.0 & 21.17 & 39.58 & 63.23 & 84.39 \\
\hline \multirow{3}{*}{$d=1$} & $1 \%$ & 0.00 & 0.01 & 0.03 & 0.21 & 6.88 & 3.20 & 9.39 & 2291 & 46.23 \\
\hline & $5 \% 6$ & 0.05 & 0,08 & 0.45 & 1. 66 & 4.87 & 12.36 & 26. 10 & 48.50 & 73.47 \\
\hline & $10 \%$ & 0.17 & 0.46 & 1. 28 & 3.97 & 9.98 & 21.63 & 39.24 & 63.69 & 84.99 \\
\hline
\end{tabular}

\begin{tabular}{|c|c|c|c|c|c|c|c|c|c|c|}
\hline True vakis of of & $\pi / \delta$ & 0.4 & 0.3 & 0.2 & 0.1 & 0 & -0.1 & -0.2 & -0.3 & -0.4 \\
\hline \multirow{3}{*}{$d=0$} & $1 \%$ & 0.00 & 0.00 & 0.01 & 0.08 & 0.99 & 7.99 & 30.02 & 68.00 & 95.48 \\
\hline & $5 \%$ & 0.00 & 0.01 & 0.05 & 0.71 & 5.32 & 19.98 & 52.53 & 85,00 & 99.16 \\
\hline & $10 \mathrm{sb}$ & 0.00 & 0.02 & 0.27 & 213 & 10.51 & 30.62 & 64.77 & 9295 & 99.87 \\
\hline \multirow{3}{*}{$d=0.5$} & $1 \%$ & 0.00 & 0.02 & 0.20 & 0,09 & 0.82 & 7,30 & 30.06 & 68.54 & 95.60 \\
\hline & $3 \%$ & 0.00 & 0.00 & 0.03 & 0.73 & 4.56 & 20.35 & 52.40 & 55,00 & 99.39 \\
\hline & $10 \%$ & 0.00 & 0.00 & 0.00 & 2.34 & 9.51 & $31+4$ & 65.10 & 92.50 & 99.89 \\
\hline \multirow{3}{*}{$d=1$} & $1 \%$ & 0.00 & 0.00 & 0.00 & 0,08 & 1. 11 & 7.71 & 2990 & 68.36 & 95.39 \\
\hline & $5 \%$ & 0.00 & 0.00 & 0.04 & 0.68 & 5.13 & $20-76$ & 51.77 & 86.05 & 99.35 \\
\hline & $10 \%$ & 0.02 & 99.97 & 0.20 & 2.09 & 9.85 & 31.71 & 64.29 & 92.41 & 99.87 \\
\hline
\end{tabular}




\section{Application to the Nelson-Plosser data}

For the sake of illustration, this section applies our $F-D F$ test to the well-known Nelson-Plosser data. The starting date is 1860 for the consumer price index and industrial production, 1869 for velocity, 1871 for stock prices, 1889 for GNP deflator and money stock, 1890 for employment and unemployment rate, 1900 for bond yield, real wages and wages, and 1909 for the nominal and real GNP and GNP per capita. The variables are expressed in natural logarithms. All variables exhibit an upward trend with the exception of velocity, which shows a strong downward trend and the unemployment rate which tends to fluctuate around a constant level. The seminal empirical work by Nelson and Plosser [18] suggests that there is a strong evidence for the unit root hypothesis for most macroeconomic time series data. Two possible specifications for the data generating processes $(D G P)$ are then

$$
y_{t}=(1-L)^{-d} u_{t}
$$

and

$$
y_{t}=\alpha+(1-L)^{-d} u_{t}
$$

The theoretical framework provided in this paper does not allow us to use the $D G P$ (5.2) (see Appendix 2). At this level, we only use the $D G P$ (5.1). For the $D G P(5.1)$, we test the null for several values of $d_{0}$, namely: $0 ; 0.5 ; 1 ; 1.5$ and 2 by using respectively the following regression models,

$$
\begin{gathered}
y_{t}=\rho \Delta^{-1} y_{t-1}+\varepsilon_{1, t}, \\
\Delta^{0.5} y_{t}=\rho \Delta^{-0.5} y_{t-1}+\varepsilon_{2, t}, \\
\Delta y_{t}=\rho y_{t-1}+\varepsilon_{3, t}, \\
\Delta^{1.5} y_{t}=\rho \Delta^{0.5} y_{t-1}+\varepsilon_{4, t}, \\
\Delta^{2} y_{t}=\rho \Delta y_{t-1}+\varepsilon_{5, t} .
\end{gathered}
$$

Note that, the sample sizes for all the 14 U.S. macroeconomic Nelson-Plosser series, used here, are between $n=80$ and $n=129$. Consequently, the decision rules adopted for the testing problem (2.3) are

$$
\begin{aligned}
& \text { reject } H_{0} \text { if } Z_{1}<-7.9, \\
& \text { reject } H_{0} \text { if } Z_{2}<-1.95,
\end{aligned}
$$

where $Z_{1}$ and $Z_{2}$ are respectively the usual statistic $n \widehat{\rho}$ and $\frac{\widehat{\rho}}{\sigma_{\widehat{\rho}}}$, and where $(-7.9,-1.95)$ are the corresponding critical values at level $\alpha=5 \%$, obtained from the usual statistical tables of Dickey-Fuller [5]. The results of the decision rules shown in Table 5 suggest that:

- for model $(I)$, all series are found to be integrated with order $d \geq 0$, 
- for model $(I I)$, all series are found to be integrated with order $d \geq 0.5$,

- for model $(I I I)$, all series are found to be integrated with order $d \geq 1$,

- for model $(I V)$, all series are found to be integrated with order $d<1.5$, except the Industrial production and Money stock series.

- for model $(V)$, all series are found to be integrated with order $d<2$.

In summary, it may be concluded from Table 5 that, following our test, all the macroeconomic variables are $d$-integrated with $1 \leq d<1.5$, except for the Industrial production and Money stock whose order of integration is between 1.5 and 2 , i.e. $1.5 \leq d<2$.

\begin{tabular}{|c|c|c|c|c|c|c|}
\hline \multirow[t]{2}{*}{ Variables (size sample) } & \multirow[t]{2}{*}{ Statistics } & \multicolumn{5}{|c|}{$d_{0}$} \\
\hline & & 0 & 0.5 & 1 & 1.5 & 2 \\
\hline \multirow{2}{*}{ Bond yeld ( 89 ) } & $z_{2}$ & 21.50 & 10. 14 & 123 & -4.89 & -16.49 \\
\hline & $z_{1}$ & 180 & 1.47 & 0.62 & $-16,71$ & $-84,96$ \\
\hline \multirow{2}{*}{ Nominal Gre? (80) } & $z_{2}$ & 19.12 & 10.52 & 6.59 & -8.18 & -95.87 \\
\hline & $z_{1}$ & 1.65 & 1.18 & 0.40 & -15.81 & -78.16 \\
\hline \multirow{2}{*}{ Reat GIVP $(30)$} & $z_{2}$ & 19.33 & 10.58 & +5 & $-7,98$ & -73.2 \\
\hline & $z_{1}$ & 1.66 & 1. 18 & 0.15 & -15.66 & -78.01 \\
\hline \multirow{2}{*}{ Real per capita CAIP (B0) } & $z_{2}$ & $17-11$ & 8. 71 & 254 & -9.63 & $-117,72$ \\
\hline & $z_{i}$ & 1.56 & 1.07 & 0.17 & -18.75 & $-78,75$ \\
\hline \multirow{2}{*}{ Real wages (89) } & $Z_{1}$ & 20.23 & 10.67 & 3.64 & -8.44 & -76.58 \\
\hline & $z_{3}$ & 8. 70 & 116 & 0,33 & -17.11 & -86.16 \\
\hline \multirow{2}{*}{ Stock prices (118) } & $Z_{1}$ & 41.95 & 18.34 & 2.49 & -3.49 & -12.52 \\
\hline & $z_{3}$ & 221 & 1.90 & 138 & -14.70 & -99.59 \\
\hline \multirow{2}{*}{ Unemploymant (99) } & $z_{2}$ & 13.85 & 2.64 & -1.12 & -5.64 & -9.3 \\
\hline & $z_{1}$ & 1.9 & 0.96 & -2.64 & -44.93 & $-89,76$ \\
\hline \multirow{2}{*}{ Veiocity (120) } & $z_{2}$ & 10.43 & 3.07 & -2.54 & -12.9 & -29.55 \\
\hline & $z_{1}$ & 116 & 0.67 & -201 & $-47,45$ & -121.66 \\
\hline \multirow{2}{*}{ Nominal wages (89) } & $z_{2}$ & 2133 & 11.93 & 6.59 & -7.9 & -80.59 \\
\hline & $z_{1}$ & 1.70 & 1.24 & 0.50 & -15.96 & -86.56 \\
\hline \multirow{2}{*}{ GNP deflator (100) } & $z_{7}$ & 24.84 & 14.47 & 5.95 & -7.57 & -56.8 \\
\hline & $z_{1}$ & 1.78 & 136 & 0,65 & -17.21 & $-97,76$ \\
\hline \multirow{2}{*}{ CPI(129) } & $z ;$ & 26.85 & 1369 & 4.53 & $-8: 73$ & -54.61 \\
\hline & $z_{1}$ & 1.74 & 136 & 0,71 & -21.89 & -12453 \\
\hline \multirow{2}{*}{ hd production (129) } & $z_{2}$ & 79.99 & 228 & 360 & -1.32 & $\sim 9,31$ \\
\hline & $z_{1}$ & 2.45 & 1.93 & 133 & -4.13 & -103.55 \\
\hline \multirow{2}{*}{ Employment (199) } & $z_{2}$ & 18.48 & 9.15 & 4.67 & -10.56 & -256.41 \\
\hline & $z_{1}$ & 154 & 1.04 & 1.50 & -22.60 & -97.84 \\
\hline \multirow{2}{*}{ Monsy stock $(100)$} & $z_{2}$ & 34.83 & 23.88 & 9.98 & -1.65 & -14.82 \\
\hline & $z_{1}$ & 2.17 & 1.75 & 131 & -1.98 & -69.20 \\
\hline
\end{tabular}

Note that the $F-D F$ test was done assuming that the empirical variables are derived from data generating process $\operatorname{ARFIMA}(0, d, 0)$. A more general 
study is needed to achieve adequate conclusions about the integration order for the Nelson-Plosser Data, by considering more general data generating process $A R F I M A(p, d, q)$ and also by incorporating non zero drift and time trend in data generating process (2.1) while using a suitable auxiliary regression model. 


\section{Concluding remarks and discussion.}

In the Dickey-Fuller paper, the parameter $d$, without restrict the generality, can have only two values $d=1$ or $d=0$. To test

$$
H_{0}: d=1 \quad \text { against } \quad H_{1}: d=0, \quad \text { (Standard test) }
$$

in the simple case, Dickey and Fuller use the regression model

$$
y_{t}=\phi y_{t-1}+\varepsilon_{t} . \quad \text { (Standard regression model) }
$$

Since Anderson [2], White ([25], [26]) developed the statistical theory on the first order autoregressive process with the autoregressive parameter equal to $1($ i.e. $d=1)$ and greater than one (explosive process), Box and Jenkins [5] formalized the analysis of time series, and Nelson and Plosser [19] argue that the most macroeconomic series have unit roots. The unit root test has been an important topic on the econometric literatures.

Phillips [20] show that under the null hypothesis (i.e. $H_{0}: d=1$ ) that the asymptotic distributions of $n(\widehat{\phi}-1)$ and $t_{\widehat{\rho}}$ are respectively,

$\begin{array}{lc}\frac{0.5\left\{W^{2}(1)-1\right\}}{\int_{0}^{1} W^{2}(r) d r} \text { and } & \frac{0.5\left\{W^{2}(1)-1\right\}}{\left[\int_{0}^{1} W^{2}(r) d r\right]^{0.5}} \\ \text { Usual asymptotic distributions for DF test }\end{array}$

where $W(\cdot)$ is the standard Brownien motion. These later asymptotic distributions has been tabulated, the tabulated values are used to perform the standard test.

In fractional case the parameter $d$ can have an infinite values, for example $d$ can have an infinite number of values $\cdots-0.5 ;-0.1 ;-0.3 ;-0.4 ;-0.5 ; 0 ; 0.1$; $0.2 ; 0.3 ; 0.4 ; 0.5 ; \cdots$. For the fractional case, the standard regression model can be used only for testing the hypothesis $H_{0}: d=1$. In our paper, the question is

\section{How to extend the standard framework above to take into account the fractional case?}

Such extension has already been discussed by Dolado et $\mathrm{Al}$ [10]. Dolado et al [10] propose to test

$$
H_{0}: d=d_{0} \text { against } \quad H_{1}: d=d_{1},
$$

by using the auxiliary regression model

$$
\Delta^{d_{0}} y_{t}=\rho \Delta^{d_{1}} y_{t-1}+\varepsilon_{t} . \quad \text { (DGM regression model) }
$$

In this paper, we show in the first step, that the $D G M$ approach is not the best and adequate way to extend the Dickey-Fuller test by taking into account the fractional case, because the $D G M$ regression model is based on the null and the alternative (i.e. $d_{0}$ and $d_{1}$ ). 
In the second step, we provide how to extend adequately the standard Dickey-Fuller test [7] by taking into account the fractional case. In fact, in our approach, the question is

\section{How to extend the standard framework below to take into account the fractional case by using the usual asymptotic distribution

$$
\frac{0.5\left\{W^{2}(1)-1\right\}}{\int_{0}^{1} W^{2}(r) d r} \text { and } \frac{0.5\left\{W^{2}(1)-1\right\}}{\left[\int_{0}^{1} W^{2}(r) d r\right]^{0.5}} ?
$$

A correct answer to this question can be very useful in practice. The answer we give to this question is based on four points:

1. Using the composite hypothesis $H_{0}: d \geq d_{0}$.

2. If $y_{t} \rightsquigarrow I\left(d_{0}\right)$ than $(1-L)^{-1+d_{0}} y_{t} \rightsquigarrow I(1)$.

3. Testing the composite null hypothesis is based upon testing the statistical significance of the coefficient $\phi$ (or $\rho=\phi-1$ ) in the regression model $\Delta^{-1+d_{0}} y_{t}=\phi \Delta^{-1+d_{0}} y_{t-1}+\varepsilon_{t}$.

4. The level of the test $\alpha=\operatorname{Sup}_{d \geq d_{0}} P\left(\right.$ reject $\left.H_{0}\right)=P\left(\right.$ reject $\left.H_{0} \mid d=d_{0}\right)$.

Our test is based on a composite null hypothesis, $H_{0}: d \geq d_{0}$, this choice was not done arbitrarily. This choice was made based on the results of the asymptotic theory given in the theorem 4 . To use our test, we recommend to follow the following steps:

1. Estimate the parameter $\rho$ in the regression model $\Delta^{d_{0}} y_{t}=\rho \Delta^{-1+d_{0}} y_{t-1}+$ $\varepsilon_{t}$. This regression provides a more flexible and unified framework to test the null for different values of $d_{0}$ while using the same critical value.

2. The null hypothesis is rejected if $Z_{i}<c_{i}(\alpha)$, where $Z_{1}=t_{\widehat{\rho}_{n}}$ and $Z_{2}=$ $n \widehat{\rho}_{n}$. The level of the test can be approximated by its asymptotic value: $\alpha=\operatorname{Sup}_{d \geq d_{0}} P\left(Z_{i}<c_{i}(\alpha)\right)=P\left[Z_{i}<c_{i}(\alpha) / d=d_{0}\right](i=1,2)$.

3 . The critical values $c_{i}(\alpha)(i=1,2)$ can be chosen so as to achieve a predetermined size by using the usual Dickey-Fuller statistical tables.

Finally, some remarks are in order:

- To implement our test we do not need to estimate the parameter $d$.

- We have referred to our test as the Fractional Dickey-Fuller $(F-D F)$ test. A similar designation, $F-D F$, has been adopted by Dolado et al [10] for their test.

- Regarding the Dickey-Pantula test, both the upward and downward procedures are still valid in our fractional case (see Dickey and Pantula [8]). Moreover, by sequentially repeating the test in upward or in the downward senses, we can cover the value of $d$ at the desired accuracy.

- The empirical study on the Nelson-Plosser Data is only made to illustrate the $F-D F$ test. 
- In this article we have not discussed the situation when there is an additional short memory component in the series, like the $A R$ or $M A$. Also, the situation when there is a non-zero drift or a time trend in data generating process may be investigated. In fact, the proposed $F-D F$ test may be easily generalized to such situations. Here, we give just an indication when $y_{t} \sim \operatorname{ARFIMA}(p, d, 0)$

$$
A(L) \Delta^{d} y_{t}=u_{t}
$$

where $A(L)=\sum_{j=0}^{p} \alpha_{j} L^{j}, L$ is the backward shift operator, $\alpha_{0}=1$, the roots of $A(z)=0$ are outside the unit circle and $u_{t}$ is defined as above. Then the fractional augmented Dickey-Fuller test, for the null hypothesis $d \geq d_{0}$, would be based on the regression model

$$
\Delta^{d_{0}} y_{t}=\rho \Delta^{-1+d_{0}} y_{t-1}+\sum_{j=0}^{p} \alpha_{j} \Delta^{d_{0}} y_{t-j}+\varepsilon_{t} .
$$

Further research is currently being undertaken toward generalizing the $F-D F$ testing approach, along similar directions as the $D F$ test has been extended in the unit root literature accounting for time series which may exhibit a trending behavior and for general ARFIMA case.

\section{References}

[1] Agiakloglou, C. and Newbold, P. Lagrange multplier tests for fractional difference, Journal of Time Series Analysis, (15), 253-262, (1994).

[2] Anderson, T.W. On the asymptotic distribution of estimates of parameters of stochastic difference equations, Ann.Math.Statist.,30, 676-687, (1959).

[3] Bensalma, A. A consistent against for unit root against fractional alternative, Fothcoming in International Journal of operational research, Inderscience Editor (2015).

[4] Bensalma, A. Unified theoretical framework for the unit root and fractional unit root, (2013), arXiv:1209.1031v2.

[5] Box, G.E.P., and G.M. Jenkins, Time series analysis: Forecasting and Control, Second Edition, Holden-Day, San Francisco, (1976).

[6] Candelon, B. and Gil-Alana. On finite Sample properties of the tests of Robinson (1994) for fractional integration, Journal of Statistical Computation and Simulation 73 (2), 445-464, (2003).

[7] Dickey, D.A. and Fuller, W.A. Distribution of the estimators for autoregressive time series with a unit root, Journal of the American Statistical Association 74 (366a), 427-431, (1979). 
[8] Dickey, D.A. and Pantula, S.G. Determining the order of differencing in autoregressive processes, Journal of Business and Economic Statistics 15 (4), 455-461, (1987).

[9] Diebold, F.X., and Rudebush, G.D. On the power of the Dickey-Fuller test against fractional alternatives, Economic Letters 35 (2), 155-160, (1991).

[10] Dolado, J.J., Gonzalo, J. and Mayoral, M. A fractional Dickey-Fuller test for unit root, Econometrica 70 (5), 1963-2006, (2002).

[11] Granger, C.W.J. and Joyeux, R. An introduction to long memory time series models and fractional differencing, Journal of Time Series Analysis 1 (1), 15-29, (1980)

[12] Hassler, U., Wolters, J. On the power of unit roots against fractionallyintegrated alternatives, Economic Letters 45 (1), 1-5, (1994).

[13] Hosking, J.R.M. Fractional differencing, Biométrika, 68 (1) 165-176, (1981).

[14] Krämer, W. Fractional integration and the augmented Dickey-Fuller test, Economics Letters 61, 269-272, (1998).

[15] Liu, M. Asymptotics of nonstationary fractional integrated series, Econometric Theory 14 (5), 641-662, (1998).

[16] Lobato, I.N. and Velasco, C. Optimal fractional Dickey-Fuller tests, Econometrics Journal 9 (3), 492-510, (2006).

[17] Lobato, I.N. and Velasco, C. Efficient Wald tests for fractional unit roots, Econometrica 75 (2), 575-589, (2007).

[18] Nelson, C.R. and Plosser, C.I. Trends and random walks in macroeconomic time series: some evidence and implications, Journal of Monetary Economics, 10 (2), 139-162, (1982).

[19] Philips, P.C.B., Time series regression with a unit root, Econometrica 55, 277-301, (1987).

[20] Robinson, P. M. Testing for strong serial correlation and dynamic conditional heteroskedasticity in multiple regression, Journal of Econometrics 47 67-84, (1991)

[21] Robinson, P.M. Efficient tests of nonstationary hypotheses, Journal of the American Statistical Association 89 (428), 1420-1437, (1994).

[22] Said, S.E., and Dickey, D.A., Testing for unit roots in autoregressive moving average models of unknown order, Biometrika, 71, 3,pp. 599-607, (1984).

[23] Sowell, F.B. The fractional unit root distribution, Econometrica, 58 (2), 494-505, (1990). 
[24] Tanaka, K. The nonstationary fractional unit root. Econometric Theory, 15 (4), 549-582, (1999).

[25] White, J.S., The limiting distribution of the serial correlation coefficient in the explosive case, Ann. Math. Statist, 29,1188-1197, (1958).

[26] White, J.S., The limiting distribution of the serial correlation coefficient in the explosive case II, Ann. Math. Statist, 30,831-384, (1959). 


\section{Appendix 1: Proof of Theorem 1}

By denoting $\Delta^{-1+d_{0}} y_{t}=x_{t}$, the $O L S$ estimator of $\rho$ and its $t$-ratio for the auxiliary regression model (3.3), are given by the usual squares expressions

$$
\widehat{\rho}_{n}=\frac{\sum_{t=1}^{n}\left(\Delta x_{t}\right)\left(x_{t-1}\right)}{\sum_{t=1}^{n}\left(x_{t-1}\right)^{2}}, \quad t_{\widehat{\rho}_{n}}=\frac{\sum_{t=1}^{n}\left(\Delta x_{t}\right)\left(x_{t-1}\right)}{\left\{\widehat{\sigma}_{n}^{2} \sum_{t=1}^{n}\left(x_{t-1}\right)^{2}\right\}^{1 / 2}}
$$

where the variance of the residuals, $\widehat{\sigma}_{n}^{2}$ is given by $\widehat{\sigma}_{n}^{2}=n^{-1} \sum_{t=1}^{n}\left(\Delta x_{t}-\widehat{\rho}_{n} x_{t-1}\right)^{2}$. Note that, $x_{t-1} \sim F I\left(1+d-d_{0}\right)$ and $\Delta x_{t} \sim F I\left(d-d_{0}\right)$. Since $x_{t}$ is stationary fractionally integrated process for $d-d_{0} \in[-1 ;-0.5[$ and nonstationary fractional integrated process for $d-d_{0} \in[-0.5 ;+\infty[$, we divide our proof into two parts

\section{Part $1: \mathbf{d}-\mathbf{d}_{0} \in[-1 ;-0.5[$}

When $-1 \leq d-d_{0}<-0.5$, given that $x_{t}$ is stationary fractionally integrated, of order $\delta \in[0 ; 0.5[$ and ergodic process, then

$$
\widehat{\rho}_{n}=\frac{\sum_{t=1}^{n}\left(\Delta x_{t}\right)\left(x_{t-1}\right)}{\sum_{t=1}^{n}\left(x_{t-1}\right)^{2}}=\frac{n^{-1} \sum_{t=1}^{n} x_{t} x_{t-1}}{n^{-1} \sum_{t=1}^{n} x_{t-1}^{2}}-1 \stackrel{P}{\rightarrow} \frac{E\left(x_{t} x_{t-1}\right)}{E\left(x_{t-1}^{2}\right)}-1 .
$$

Therefore, given that

$$
E\left(x_{t} x_{t-j}\right)=\sigma_{u}^{2} \frac{\Gamma(j+\delta) \Gamma(1-2 \delta)}{\Gamma(j+1-\delta) \Gamma(1-\delta) \Gamma(\delta)}, \quad j \geq 0,
$$

(see, Hosking [12]) and the recursive identity $\Gamma(1+z)=z \Gamma(z)$, it follows that

$$
\widehat{\rho}_{n} \stackrel{P}{\rightarrow} \frac{E\left(x_{t} x_{t-1}\right)}{E\left(x_{t-1}^{2}\right)}=-\frac{1}{1-\delta},
$$

which, in turn, given that $\delta \in\left[0 ; 0.5\left[\right.\right.$, entails that $\widehat{\rho}_{n} \in[-2 ;-1[$. Consequently, $n \widehat{\rho}_{n} \stackrel{P}{\rightarrow}-\infty$. With respect to the $t$-test (i.e. $t_{\widehat{\rho}_{n}}$ ), it is straightforward to prove that

$$
n^{-1} \widehat{\sigma}_{n}^{2} \sum_{t=1}^{n}\left(x_{t-1}\right)^{2}=n^{-2}\left(\left(\sum_{t=1}^{n} x_{t}^{2}\right)\left(\sum_{t=1}^{n} x_{t-1}^{2}\right)-\left(\sum_{t=1}^{n} x_{t} x_{t-1}\right)^{2}\right),
$$

and then, by using $\left(A_{0}\right)$ and the recursive identity $\Gamma(1+z)=z \Gamma(z)$, it follows that

$$
\left(n^{-1} \widehat{\sigma}_{n}^{2} \sum_{t=1}^{n}\left(x_{t-1}\right)^{2}\right)^{\frac{1}{2}} \stackrel{p}{\rightarrow} \sigma_{u}^{2} \frac{\Gamma(1-2 \delta)}{\Gamma(1-\delta)^{2}} \frac{\sqrt{1-2 \delta}}{1-\delta},
$$

and

$$
n^{-1}\left(\sum_{t=1}^{n} x_{t} x_{t-1}-\sum_{t=1}^{n} x_{t-1}^{2}\right) \stackrel{p}{\rightarrow} \sigma_{u}^{2} \frac{\Gamma(1-2 \delta)}{\Gamma(1-\delta)^{2}} \frac{2 \delta-1}{1-\delta} .
$$

Consequently, $n^{-1 / 2} t_{\widehat{\rho}_{n}} \rightarrow-\sqrt{1-2 \delta}$ and then $t_{\widehat{\rho}_{n}} \stackrel{p}{\rightarrow}-\infty$. 


\section{Part 2: $\mathbf{d}-\mathbf{d}_{\mathbf{0}} \in[-0.5 ;+\infty[$.}

For the $\sum_{t=1}^{n}\left(x_{t-1}\right)^{2}$ term, it follows from (3.4), (3.5), (3.6), (3.7) and the continuous mapping theorem. When $d-d_{0}=-0.5$,

$$
\frac{1}{n(\log n) \kappa_{\eta}^{2}\left(\frac{1}{2}\right)} \sum_{t=1}^{n}\left(x_{t-1}\right)^{2} \Rightarrow \int_{0}^{1} \mathbf{w}_{0.5}^{2}(r) d r .
$$

When $-\frac{1}{2}<d-d_{0}<0$,

$$
\frac{1}{n^{2+2 \delta} \kappa_{\eta}^{2}(\delta)} \sum_{t=1}^{n}\left(x_{t-1}\right)^{2} \Rightarrow \int_{0}^{1} \mathbf{w}_{\delta}^{2}(r) d r
$$

When $d-d_{0}=0$,

$$
\frac{1}{n^{2} \kappa_{\eta}^{2}(0)} \sum_{t=1}^{n}\left(x_{t-1}\right)^{2} \Rightarrow \int_{0}^{1} \mathbf{w}^{2}(r) d r .
$$

When $0<d-d_{0}<\frac{1}{2}$,

$$
\frac{1}{n^{2+2 \delta} \kappa_{\eta}^{2}(\delta)} \sum_{t=1}^{n}\left(x_{t-1}\right)^{2} \Rightarrow \int_{0}^{1} \mathbf{w}_{\delta}^{2}(r) d r
$$

When $d-d_{0}=m+\frac{1}{2}, m \geq 1$,

$$
\frac{1}{n^{2\left(m+\frac{3}{2}\right)}(\log n) \kappa_{\eta}^{2}\left(\frac{1}{2}\right)} \sum_{t=1}^{n}\left(x_{t-1}\right)^{2} \Rightarrow \int_{0}^{1} \mathbf{w}_{0.5, m+1}^{2}(r) d r .
$$

When $d-d_{0}=m+\delta, m \geq 1$,

$$
\frac{1}{n^{2(m+1+\delta)} \kappa_{\eta}^{2}(\delta)} \sum_{t=1}^{n}\left(x_{t-1}\right)^{2} \Rightarrow \int_{0}^{1} \mathbf{w}_{\delta, m+1}^{2}(r) d r
$$

For the $\sum_{t=1}^{n}\left[\Delta x_{t}\right]\left[x_{t-1}\right]$ term, we have

$$
\sum_{t=1}^{n}\left[\Delta x_{t}\right]\left[x_{t-1}\right]=\frac{1}{2}\left(\Delta^{-1+d_{0}} y_{n}\right)^{2}-\frac{1}{2} \sum_{t=1}^{n}\left(\Delta x_{t}\right)^{2}
$$

For the first term, it follows from (3.4), (3.5), (3.6), (3.7) and the continuous mapping theorem. When $d-d_{0}=-0.5$,

$$
\frac{1}{2(\log n) \kappa_{\eta}^{2}\left(\frac{1}{2}\right)}\left(\Delta^{-1+d_{0}} y_{n}\right)^{2} \Rightarrow \frac{1}{2} \mathbf{w}_{0.5}^{2}(1)
$$

When $-0.5<d-d_{0}<0$,

$$
\frac{1}{2 n^{1+2 \delta} \kappa_{\eta}^{2}(\delta)}\left(\Delta^{-1+d_{0}} y_{n}\right)^{2} \Rightarrow \frac{1}{2} \mathbf{w}_{\delta}^{2}(1)
$$


When $d-d_{0}=0$,

$$
\frac{1}{2 n^{1} \kappa_{\eta}^{2}(0)}\left(\Delta^{-1+d_{0}} y_{n}\right)^{2} \Rightarrow \frac{1}{2} \mathbf{w}^{2}(1) .
$$

When $0<d-d_{0}<0.5$,

$$
\frac{1}{2 n^{1+2 \delta} \kappa_{\eta}^{2}(\delta)}\left(\Delta^{-1+d_{0}} y_{n}\right)^{2} \Rightarrow \frac{1}{2} \mathbf{w}_{\delta}^{2}(1) .
$$

When $d-d_{0}=m+0.5, m \geq 1$,

$$
\frac{1}{2 n^{2(m+1)}(\log n) \kappa_{\eta}^{2}\left(\frac{1}{2}\right)}\left(\Delta^{-1+d_{0}} y_{n}\right)^{2} \Rightarrow \frac{1}{2} \mathbf{w}_{0.5, m+1}^{2}(1) .
$$

when $d-d_{0}=m+\delta, m \geq 1$,

$$
\frac{1}{2 n^{1+2(m+\delta)} \kappa_{\eta}^{2}\left(\frac{1}{2}\right)}\left(\Delta^{-1+d_{0}} y_{n}\right)^{2} \Rightarrow \frac{1}{2} \mathbf{w}_{\delta, m+1}^{2}(1) .
$$

For the second term, we have:

When $d-d_{0}=-0.5$, by using Lemma 2.1 of Ming Liu (1998) result 2

$$
-\frac{1}{2} \kappa_{\eta}^{-2}\left(\frac{1}{2}\right) n^{-1} \sum_{t=1}^{n}\left(\Delta x_{t}\right)^{2} \stackrel{p}{\rightarrow}-\frac{1}{2} \kappa_{\eta}^{-2}\left(\frac{1}{2}\right) \operatorname{var}\left(\Delta x_{t}\right)=-1 .
$$

When $-0.5<d-d_{0}<0$, by using (3.4) and the ergodic theorem (note that here $d-d_{0}=\delta$ )

$$
-\frac{1}{2} \kappa_{\eta}^{-2}(\delta) n^{-1} \sum_{t=1}^{n}\left(\Delta x_{t}\right)^{2} \stackrel{p}{\rightarrow}-\frac{1}{2} \kappa_{\eta}^{-2}(\delta) \operatorname{var}\left(\Delta x_{t}\right)=-\left(\frac{1}{2}+\delta\right) \frac{\Gamma(1+\delta)}{\Gamma(1-\delta)} .
$$

When $d-d_{0}=0$, by using (3.4) and the ergodic theorem

$$
-\frac{1}{2} \kappa_{\eta}^{-2}(0) n^{-1} \sum_{t=1}^{n}\left(\Delta x_{t}\right)^{2} \stackrel{p}{\rightarrow}-\frac{1}{2} \kappa_{\eta}^{-2}(0) \operatorname{var}\left(\Delta x_{t}\right)=-\frac{1}{2} .
$$

When $0<d-d_{0}<0.5$, by using (3.4) and the ergodic theorem (note that here $\left.d-d_{0}=\delta\right)$

$$
-\frac{1}{2} n^{-1} \sum_{t=1}^{n}\left(\Delta x_{t}\right)^{2} \stackrel{p}{\rightarrow}-\frac{1}{2} \kappa_{\eta}^{-2}(\delta) \operatorname{var}\left(\Delta x_{t}\right)=-\left(\frac{1}{2}+\delta\right) \frac{\Gamma(1+\delta)}{\Gamma(1-\delta)} .
$$

When $d-d_{0}=m+0.5, m \geq 1$, by using (3.5), (3.7) and the continuous mapping theorem

$$
\frac{1}{n^{2(m+0.5)}(\log n) \kappa_{\eta}^{2}(0.5)} \sum_{t=1}^{n}\left(\Delta x_{t}\right)^{2} \Rightarrow \int_{0}^{1} \mathbf{w}_{0.5, m}^{2}(r) d r .
$$


When $d-d_{0}=m+\delta, m \geq 1$, by using (3.4), (3.6) and the continuous mapping theorem

$$
\frac{1}{n^{2(m+\delta)} \kappa_{\eta}^{2}(\delta)} \sum_{t=1}^{n}\left(\Delta x_{t}\right)^{2} \Rightarrow \int_{0}^{1} \mathbf{w}_{\delta, m}^{2}(r) d r .
$$

Therefore, when $d-d_{0}=-0.5$, we have, using $(A 7)$ and $(A 13)$,

$$
n^{-1} \kappa_{\eta}^{-2}(0.5) \sum_{t=1}^{n}\left[\Delta x_{t}\right]\left[x_{t-1}\right] \stackrel{p}{\rightarrow}-1 .
$$

When $-0.5<d-d_{0}<0$, by using $(A 8)$ and $(A 14)$, we have

$$
n^{-1} \kappa_{\eta}^{-2}(\delta) \sum_{t=1}^{n}\left[\Delta x_{t}\right]\left[x_{t-1}\right] \stackrel{p}{\rightarrow}-\left(\frac{1}{2}+\delta\right) \frac{\Gamma(1+\delta)}{\Gamma(1-\delta)} .
$$

When $d-d_{0}=0$, by using $(A 9)$ and $(A 15)$, we have

$$
n^{-1} \kappa_{\eta}^{-2}(0) \sum_{t=1}^{n}\left[\Delta x_{t}\right]\left[x_{t-1}\right] \Rightarrow-\frac{1}{2}\left\{\mathbf{w}^{2}(1)-1\right\} .
$$

When $0<d-d_{0}<0.5$, by using $(A 10)$ and $(A 16)$, we have

$$
n^{-1-2 \delta} \kappa_{\eta}^{-2}(\delta) \sum_{t=1}^{n}\left[\Delta x_{t}\right]\left[x_{t-1}\right] \Rightarrow \frac{1}{2} \mathbf{w}_{\delta}^{2}(1) .
$$

When $d-d_{0}=m+0.5, m \geq 1$, by using $(A 11)$ and $(A 17)$, we have

$$
n^{-2(m+1)}\left(\log ^{-1} n\right) \kappa_{\eta}^{-2}(0.5) \sum_{t=1}^{n}\left[\Delta x_{t}\right]\left[x_{t-1}\right] \Rightarrow \frac{1}{2} \mathbf{w}_{0.5, m+1}^{2}(1) .
$$

When $d-d_{0}=m+\delta, m \geq 1$

$$
n^{-1-2(m+\delta)} \kappa_{\eta}^{-2}(\delta) \sum_{t=1}^{n}\left[\Delta x_{t}\right]\left[x_{t-1}\right] \Rightarrow \frac{1}{2} \mathbf{w}_{\delta, m+1}^{2}(1) .
$$

Hence, using respectively (A1,A19), (A2,A20), (A3,A21), (A4,A22), (A5,A23), $(\mathrm{A} 6, \mathrm{~A} 24)$ and the continuous mapping theorem, we obtain When $d-d_{0}=-0.5$,

$$
(\log n) \widehat{\rho}_{n}=\frac{n^{-1} \kappa_{\eta}^{-2}(0.5) \sum_{t=1}^{n}\left[\Delta x_{t}\right]\left[x_{t-1}\right]}{n^{-1}\left(\log ^{-1} n\right) \kappa_{\eta}^{-2}(0.5) \sum_{t=1}^{n}\left[x_{t-1}\right]^{2}} \Rightarrow \frac{-1}{\int_{0}^{1} \mathbf{w}_{0.5}^{2}(r) d r} .
$$

When $-0.5<d-d_{0}<0$,

$$
n^{1+2 \delta} \widehat{\rho}_{n}=\frac{n^{-1} \kappa_{\eta}^{-2}(\delta) \sum_{t=1}^{n}\left[\Delta x_{t}\right]\left[x_{t-1}\right]}{n^{-2-2 \delta} \kappa_{\eta}^{-2}(\delta) \sum_{t=1}^{n}\left[x_{t-1}\right]^{2}} \Rightarrow \frac{-\left(\frac{1}{2}+\delta\right) \frac{\Gamma(1+\delta)}{\Gamma(1-\delta)}}{\int_{0}^{1} \mathbf{w}_{\delta}^{2}(r) d r} .
$$


When $d-d_{0}=0$,

$$
n \widehat{\rho}_{n}=\frac{n^{-1} \kappa_{\eta}^{-2}(0) \sum_{t=1}^{n}\left[\Delta x_{t}\right]\left[x_{t-1}\right]}{n^{-2} \kappa_{\eta}^{-2}(0) \sum_{t=1}^{n}\left[x_{t-1}\right]^{2}} \Rightarrow \frac{\frac{1}{2}\left[\mathbf{w}^{2}(1)-1\right]}{\int_{0}^{1} \mathbf{w}^{2}(r) d r} .
$$

When $0<d-d_{0}<0.5$,

$$
n \widehat{\rho}_{n}=\frac{n^{-1-2 \delta} \kappa_{\eta}^{-2}(\delta) \sum_{t=1}^{n}\left[\Delta x_{t}\right]\left[x_{t-1}\right]}{n^{-2-2 \delta} \kappa_{\eta}^{-2}(\delta) \sum_{t=1}^{n}\left[x_{t-1}\right]^{2}} \Rightarrow \frac{\frac{1}{2} \mathbf{w}_{\delta}^{2}(1)}{\int_{0}^{1} \mathbf{w}_{\delta}^{2}(r) d r} .
$$

When $d-d_{0}=m+0.5, m \geq 1$,

$$
n \widehat{\rho}_{n}=\frac{n^{-2(m+1)}\left(\log ^{-1} n\right) \kappa_{\eta}^{-2}(0.5) \sum_{t=1}^{n}\left[\Delta x_{t}\right]\left[x_{t-1}\right]}{n^{-2(m+1.5)}\left(\log ^{-1} n\right) \kappa_{\eta}^{-2}(0.5) \sum_{t=1}^{n}\left[x_{t-1}\right]^{2}}
$$

and then

$$
n \widehat{\rho}_{n} \Rightarrow \frac{\frac{1}{2} \mathbf{w}_{0.5, m+1}^{2}(1)}{\int_{0}^{1} \mathbf{w}_{0.5, m+1}^{2}(r) d r} \equiv \rho_{1, \infty} .
$$

when $d-d_{0}=m+\delta, m \geq 1$,

$$
n \widehat{\rho}_{n}=\frac{n^{-2(m+\delta)} \kappa_{\eta}^{-2}(\delta) \sum_{t=1}^{n}\left[\Delta x_{t}\right]\left[x_{t-1}\right]}{n^{-2(m+1+\delta)} \kappa_{\eta}^{-2}(\delta) \sum_{t=1}^{n}\left[x_{t-1}\right]^{2}} \Rightarrow \frac{\frac{1}{2} \mathbf{w}_{\delta, m+1}^{2}(1)}{\int_{0}^{1} \mathbf{w}_{\delta, m+1}^{2}(r) d r} \equiv \rho_{2, \infty} .
$$

Now consider the $t$-statistic. First notice that

$\widehat{\sigma}_{n}^{2}=n^{-1}\left(\sum_{t=1}^{n}\left(\Delta x_{t}\right)^{2}+\widehat{\rho}_{n}^{2} \sum_{t=1}^{n}\left(x_{t-1}\right)^{2}-2 \widehat{\rho}_{n} \sum_{t=1}^{n}\left[\Delta x_{t}\right]\left[x_{t-1}\right]\right)$. Hence, when $d-d_{0}=-0.5$, by using $(A 1),(A 13)(A 19)$ and $(A 25)$, it follows

$$
\widehat{\sigma}_{n}^{2} \stackrel{p}{\rightarrow} \operatorname{var}\left(\Delta x_{t}\right)=\frac{4 \sigma_{\varepsilon}^{2}}{\pi} .
$$

When $-0.5<d-d_{0}<0$, by using $A 2, A 14, A 20$ and $A 26$, it follows

$$
\widehat{\sigma}_{n}^{2} \stackrel{p}{\rightarrow} \operatorname{var}\left(\Delta x_{t}\right)=\frac{\sigma_{\varepsilon}^{2} \Gamma(1-2 \delta)}{\Gamma^{2}(1-\delta)} .
$$

When $d-d_{0}=0$, by using $A 3, A 15, A 21$ and $A 27$, it follows

$$
\widehat{\sigma}_{n}^{2} \stackrel{p}{\rightarrow} \operatorname{var}\left(\Delta x_{t}\right)=\sigma_{\varepsilon}^{2} .
$$

When $0<d-d_{0}<0.5$, by using $A 4, A 16, A 22$ and $A 28$, it follows

$$
\widehat{\sigma}_{n}^{2} \stackrel{p}{\rightarrow} \operatorname{var}\left(\Delta x_{t}\right)=\frac{\sigma_{\varepsilon}^{2} \Gamma(1-2 \delta)}{\Gamma^{2}(1-\delta)} .
$$

When $d-d_{0}=m+0.5, m \geq 1$, by using $A 5, A 17, A 23$ and $A 29$, it follows

$$
\frac{\kappa_{\eta}^{-2}(0.5) \widehat{\sigma}_{n}^{2}}{n^{2 m}(\log n)} \Rightarrow \int_{0}^{1} \mathbf{w}_{0.5, m}^{2}(r) d r+\rho_{1, \infty}^{2} \int_{0}^{1} \mathbf{w}_{0.5, m+1}^{2}(r) d r-\rho_{1, \infty} \mathbf{w}_{0.5, m+1}^{2}(1) \text {. }
$$


When $d-d_{0}=m+\delta, m \geq 1$ by using $A 6, A 18, A 24$ and $A 30$, it follows

$n^{-2 m-2 \delta+1} \kappa_{\eta}^{-2}(\delta) \widehat{\sigma}_{n}^{2} \Rightarrow \int_{0}^{1} \mathbf{w}_{\delta, m}^{2}(r) d r+\rho_{2, \infty}^{2} \int_{0}^{1} \mathbf{w}_{\delta, m+1}^{2}(r) d r-\rho_{2, \infty} \mathbf{w}_{\delta, m+1}^{2}(1)$.

Finally, by using respectively (A1,A19,A31), (A2,A20,A32), (A3,A21,A33), (A4,A22,A34) (A5,A23,A35), (A6,A24,A36) we obtain for the $t$-statistic:

When $d-d_{0}=-0.5, t_{\widehat{\rho}_{n}}=\frac{n^{-0.5}\left(\log ^{-0.5} n\right) \kappa_{\eta}^{-1}(0.5) \sum_{t=1}^{n}\left[\Delta x_{t}\right]\left[x_{t-1}\right]}{\widehat{\sigma}_{n}\left\{n^{-1}\left(\log ^{-1} n\right) \kappa_{\eta}^{-2}(0.5) \sum_{t=1}^{n}\left[x_{t-1}\right]^{2}\right\}^{0.5}} \stackrel{p}{\rightarrow}-\infty$, and $t_{\widehat{\rho}_{n}}=O_{p}\left(n^{0.5} \log ^{0.5} n\right)$.

When $-0.5<d-d_{0}<0, t_{\widehat{\rho}_{n}}=\frac{n^{-\delta-1} \kappa_{\eta}^{-1}(\delta) \sum_{t=1}^{n}\left[\Delta x_{t}\right]\left[x_{t-1}\right]}{\widehat{\sigma}_{n}\left\{n^{-2 \delta-2} \kappa_{\eta}^{-2}(\delta) \sum_{t=1}^{n}\left[x_{t-1}\right]^{2}\right\}^{0.5}} \stackrel{p}{\rightarrow}-\infty$, and $t_{\widehat{\rho}_{n}}=O_{p}\left(n^{-\delta}\right)$.

When $d-d_{0}=0, t_{\widehat{\rho}_{n}}=\frac{n^{-1} \kappa_{\eta}^{-1}(0) \sum_{t=1}^{n}\left[\Delta x_{t}\right]\left[x_{t-1}\right]}{\widehat{\sigma}_{n}\left\{n^{-2} \kappa_{\eta}^{-2}(0) \sum_{t=1}^{n}\left[x_{t-1}\right]^{2}\right\}^{0.5}} \Rightarrow \frac{\frac{1}{2}\left[\mathbf{w}^{2}(1)-1\right]}{\left[\int_{0}^{1} \mathbf{w}^{2}(r) d r\right]^{0.5}}$, and $t_{\widehat{\rho}_{n}}=O_{p}(1)$.

When $0<d-d_{0}<0.5, t_{\widehat{\rho}_{n}}=\frac{n^{-\delta-1} \kappa_{\eta}^{-1}(\delta) \sum_{t=1}^{n}\left[\Delta x_{t}\right]\left[x_{t-1}\right]}{\widehat{\sigma}_{n}\left\{n^{-2 \delta-2} \kappa_{\eta}^{-2}(\delta) \sum_{t=1}^{n}\left[x_{t-1}\right]^{2}\right\}^{0.5}} \stackrel{p}{\rightarrow}-\infty$, and $t_{\widehat{\rho}_{n}}=O_{p}\left(n^{\delta}\right)$.

When $d-d_{0}=m+0.5, m \geq 1$,

$t_{\widehat{\rho}_{n}}=\frac{n^{0.5}\left\{n^{-2(m+1)}\left(\log ^{-1} n\right) \kappa_{\eta}^{-2}(0.5) \sum_{t=1}^{n}\left[\Delta x_{t}\right]\left[x_{t-1}\right]\right\}}{\left\{n^{-m}\left(\log ^{-0.5} n\right) \kappa_{\eta}^{-1}(0.5) \widehat{\sigma}_{n}\right\}\left\{n^{-2(m+1.5)}\left(\log ^{-1} n\right) \kappa_{\eta}^{-2}(0.5) \sum_{t=1}^{n}\left[x_{t-1}\right]^{2}\right\}^{0.5}} \stackrel{p}{\rightarrow}+\infty$, and $t_{\widehat{\rho}_{n}}=O_{p}\left(n^{0.5}\right)$.

When $d-d_{0}=m+\delta, m \geq 1$,

$t_{\widehat{\rho}_{n}}=\frac{n^{0.5}\left\{n^{-1-2(m+1)} \kappa_{\eta}^{-2}(\delta) \sum_{t=1}^{n}\left[\Delta x_{t}\right]\left[x_{t-1}\right]\right\}}{\left\{n^{-m-\delta+0.5} \kappa_{\eta}^{-1}(\delta) \widehat{\sigma}_{n}\right\}\left\{n^{-2(m+1+\delta)} \kappa_{\eta}^{-2}(\delta) \sum_{t=1}^{n}\left[x_{t-1}\right]^{2}\right\}^{0.5}} \stackrel{p}{\rightarrow}+\infty$, and $t_{\widehat{\rho}_{n}}=$ $O_{p}\left(n^{0.5}\right)$.

Appendix 2: F - DF test in the Presence of deterministic components

We assume that the univariate process $\left\{y_{t}, t \in \mathbb{Z}\right\}$ can be generated by the following two mechanisms

$$
y_{t}=\alpha+(1-L)^{-d} u_{t}, \quad t \in \mathbb{Z},
$$

and

$$
y_{t}=\alpha+\beta t+(1-L)^{-d} u_{t}, \quad t \in \mathbb{Z},
$$

where $u_{t}$ is as in (2.1). For $D G P(A)$ and $(B)$ we can use the $F-D F$ test without having to use their specific asymptotic theory. This can be done by differencing the process $y_{t}$ one time in $(A)$ and twice in $(B)$. To be more clear, we consider testing hypotheses

$$
H_{0}: d \geq d_{0} \text { against } H_{1}: d<d_{0},
$$

even though, the framework provided in this paper does not allow us to use the $D G P(A)$ and $(B)$, we can, nevertheless use it as the following.

For the $D G P(A)$, the constant, $(\alpha)$, can be removed by first differencing,

$$
(1-L) y_{t}=(1-L)^{1-d} u_{t},
$$


and then, by transforming the $D G P(A)$ in this way, instead $(C)$, we consider testing hypotheses

$$
H_{0}: d_{1} \geq d_{0}-1 \quad \text { against } \quad H_{1}: d_{1}<d_{0}-1, \quad \text { where } d_{1}=d-1,
$$

by using the auxiliary regression model

$$
\Delta^{d_{0}-1} y_{t}=\rho \Delta^{d_{0}} y_{t-1}+\varepsilon_{t} .
$$

For the $D G P(B)$, the constant, $(\alpha)$, and the parameter time trend, $(\beta)$, can be removed by differencing twice the process $y_{t}$,

$$
(1-L)^{2} y_{t}=(1-L)^{d-2} u_{t},
$$

and then, for this transformed model, instead $(C)$, we consider testing hypotheses

$$
H_{0}: d_{2} \geq d_{0}-2 \quad \text { against } \quad H_{1}: d_{2}<d_{0}-2, \quad \text { where } d_{2}=d-2,
$$

by using the auxiliary regression model

$$
\Delta^{d_{0}-2} y_{t}=\rho \Delta^{d_{0}-1} y_{t-1}+\varepsilon_{t} .
$$

